\title{
EMULSIONS STABILISED WITH POLYELECTROLYTE COMPLEXES PREPARED FROM A MIXTURE OF A WEAK AND A STRONG POLYECTROLYTE
}

\author{
Ana Maria Bago Rodriguez, ${ }^{1}$ Bernard P. Binks ${ }^{1, *}$ and Tomoko Sekine ${ }^{2}$ \\ ${ }^{1}$ Department of Chemistry and Biochemistry, University of Hull, Hull, HU6 7RX, U.K. \\ ${ }^{2}$ Shiseido Global Innovation Center, 1-2-11, Takashima, Nishi-ku, Yokohama, 220-0011, \\ Japan
}

Submitted to: $\quad$ Langmuir on 26.3.19; revised on 25.4.19

Contains ESI

*Proofs and correspondence to: $\quad$ Professor B.P. Binks

b.p.binks@hull.ac.uk 


\section{ABSTRACT}

The possibility of stabilising emulsions with polyelectrolyte complexes (PEC) obtained from the interaction of two non-surface active oppositely charged polyelectrolytes (PEL) is described. Poly(allylamine hydrochloride) (PAH) and poly(4-styrene sulfonate) sodium salt (PSSNa) are selected as the weak cationic and the strong anionic polyelectrolyte, respectively. Aqueous polymer mixtures are investigated by light scattering to determine the size of the complexes and whether precipitation or complex coacervation occurs. The effects of PEL mixing ratio, $\mathrm{pH}$ and $\mathrm{PEL}$ concentration are studied in detail. By increasing the $\mathrm{pH}$, the transition precipitate-precipitate/coacervate-coacervate-polymer solution is observed. At low $\mathrm{pH}$, both PEL are fully ionised and therefore precipitates (soft particles) arise as a result of strong electrostatic interactions. By increasing the $\mathrm{pH}$, the degree of ionisation of $\mathrm{PAH}$ decreases and weak electrostatic interactions ensue, supporting the formation of coacervate droplets. The most stable oil-in-water emulsions are prepared from aqueous mixtures around charge neutralisation. Although emulsions can be prepared from coacervate droplet dispersions their coalescence stability is worse than those stabilised by soft PEC particles. By increasing the PEL concentration, the average droplet diameter decreases and the fraction of cream in the emulsion increases for emulsions prepared with PEC particles, following the limited coalescence model. However, at high concentrations, emulsion stability is slightly worse probably due to extensive aggregation of the particles. Viscous high internal phase emulsions can be prepared at low pH in which oil droplets are deformed. Here, PEC particles are detected only at the oil-water interface. At lower oil content, excess particles form a network in the aqueous phase aiding emulsion stability to coalescence. 


\section{INTRODUCTION}

Emulsions normally contain two immiscible liquids where one is dispersed in the other as drops of microscopic or colloidal size. ${ }^{1}$ Among various applications, they are widely used in the food, ${ }^{2}$ cosmetics $^{3}$ and pharmaceutical ${ }^{4}$ industries. Due to their thermodynamic instability, addition of a surface-active material or emulsifier is required. Solid particles are one type of emulsifier, giving rise to the so-called Pickering emulsions. The discovery has been attributed to Pickering ${ }^{5}$ (1907) despite the fact that four years earlier Ramsden ${ }^{6}$ described the formation of a membrane of solid particles enveloping both air bubbles in water (foam) and oil drops in water (emulsion). The stability of emulsions by solid particles is enhanced compared to those stabilized by surfactants. Particles may become irreversibly anchored at the interface of dispersed drops forming a steric barrier that prevents or inhibits coalescence. The stabilisation mechanism in this case is explained by the particle wettability ${ }^{7}$ via the contact angle that particles make at the oil-water interface. ${ }^{8}$ Several authors have reported on emulsion stabilisation by mixtures of particles of opposite charge. ${ }^{9-12}$ Upon their interaction, aggregates of low overall charge become surface-active at the oil-water interface while emulsions cannot be prepared with either positive or negative particles as they are too hydrophilic. Despite many examples of emulsions stabilised by hard particles encountered in the literature, ${ }^{13-16}$ reports on emulsions stabilised by soft or deformable particles are not numerous. One example of this kind are emulsions stabilised by microgel particles (cross-linked polymer particles that are swollen by a solvent). ${ }^{17-22}$ Microgels, opposite to rigid particles, become deformed at the oilwater interface due to their softness. ${ }^{23}$ Another type of particle that could be included within this category are those that arise from the electrostatic interaction of two oppositely charged polyelectrolytes, which constitute a type of phase separation in polymer mixtures.

Upon mixing two aqueous polymer solutions, three different scenarios can arise as summarised by Albertsson. ${ }^{24}$ Segregative phase separation takes place when the polymers repel each other, i.e. they are incompatible. As a result each polymer is collected, predominantly, in a different phase. This phenomenon was first reported by Beijerinck in $1896 .{ }^{25}$ After mixing a solution of gelatin with either agar or soluble starch, a turbid mixture occurred which then separated into two liquid layers, with the top layer rich in gelatin and the bottom layer rich in either agar or starch. ${ }^{25,26}$ On the other hand, complete miscibility gives rise to a homogeneous solution. An example of miscibility in the macroscopic level is the mixture of polystyrene and poly(2,6dimethyl-1,4-phenylene oxide). ${ }^{27}$ Incompatibility is the most common phenomenon that occurs upon mixing two polymer solutions as observed by Dobry and Boyer-Kawenoki after a 
systematic investigation regarding the miscibility of pairs of different polymers in organic solvents. ${ }^{28}$ The third type of phase separation arises from the interaction between two polyelectrolytes. A polyelectrolyte (PEL) is a water-soluble polymer that contains numerous ionisable groups (either cationic or anionic) along the chain and low molecular weight counterions to ensure electroneutrality. ${ }^{29}$ Two oppositely charged polyelectrolytes can interact through electrostatic interactions mainly, to form the so-called polyelectrolyte complexes (PEC). As a result, the macromolecular self-assembly is collected in one phase while the other phase consists almost entirely of solvent. These two phases are immiscible and hence incompatible. However, this incompatibility occurs as a result of an associative phase separation, opposite to the segregative phase separation mentioned previously. Depending on the strength of the interaction, either a precipitate (amorphous solid particles) or a complex coacervate (micron sized droplets) can be formed. The term complex coacervate was coined by Bungenberg de Jong and Kruyt in order to distinguish it from a simple coacervate, in which one macromolecule and a dehydrating agent take part in the associative process. ${ }^{30}$ Coacervation was extensively studied by Bungenberg de Jong and co-workers for natural polyelectrolytes and they found that phase separation occurred only when gelatin was positively charged and gum Arabic was negatively charged. ${ }^{30}$ It is difficult to predict whether a precipitate (solidliquid type of phase separation) or a complex coacervate (liquid-liquid type of phase separation) will be formed. However, precipitates tend to occur when the electrostatic interactions between PEL are strong while complex coacervate formation arises when the interactions are relatively weak. ${ }^{31,32}$ However, other interactions such as hydrogen bonding, van der Waals forces or hydrophobic interactions, can play an important role in the complexation process.

In our recent publications, we introduce the idea of preparing emulsions stabilised by polyelectrolyte complexes (PEC) made from a mixture of two non-surface-active oppositely charged polyelectrolytes. As these complexes are formed mainly by the electrostatic interaction (non-covalent) of two charged polymers, the resulting complex can be considered a kind of a soft particle. The literature regarding emulsion stabilisation with complexes formed upon the interaction between a protein and a polysaccharide is extensive. ${ }^{33-36}$ However, either one or both polyelectrolytes are surface-active on their own, unlike the system described here. Two polyelectrolyte systems of this kind have been reported recently and comprise a systematic study of aqueous PEC dispersions and emulsions prepared from them. ${ }^{37,38}$ The first system consisted of the mixture between two strong polyelectrolytes: poly(diallyldimethylammonium chloride), PDADMAC and poly(4-styrene sulfonate) sodium salt, PSSNa. ${ }^{37}$ Precipitates were 
detected across the whole mole fraction range of the anionic polyelectrolyte and emulsions with higher stability were obtained around charge neutrality. ${ }^{37}$ The second system consisted of the mixture between a strong cationic and weak anionic polyelectrolyte: PDADMAC and poly(acrylic acid) sodium salt, PAANa. ${ }^{38}$ Unlike the previous system, $\mathrm{pH}$ played an important role in the strength of the interaction. As a result, both precipitates and complex coacervates were detected across the XPAANa and $\mathrm{pH}$ range. Emulsions stabilised by coacervate droplets by addition of oil stepwise and multiple homogenisation steps were prepared. Unlike the first system, emulsions could not be obtained with PEC precipitates probably due to their large size, their relatively low amount or their inherent hydrophilicity. ${ }^{38}$ In the present paper we move forward to a system composed by a weak cationic, poly(allylamine hydrochloride), PAH and a strong anionic polyelectrolyte, PSSNa. The same systematic approach was followed to evaluate the influence of parameters such as the mole fraction of the anionic polyelectrolyte, PEL concentration and $\mathrm{pH}$ for aqueous PEC dispersions. The same parameters were evaluated for emulsions together with the oil volume fraction $\left(\phi_{o}\right)$. High internal phase emulsions (HIPEs) up to $\phi_{o}=0.85$ with high viscosity were prepared with PEC particles as emulsifier. The formation of HIPEs is not common in particle-stabilised systems, since catastrophic phase inversion is usually brought about by changing the oil:water ratio. Taking advantage of the inherent fluorescence of each PEL, PEC particles could be visualised without any further modification through confocal laser scanning microscopy (CLSM). The interface of disperse drops was found to be sparsely covered by PEC particles at high $\phi_{0}$. Combining the conclusions reported here with those from previous studies, ${ }^{37,38}$ we put forward some general rules of behaviour which will help to understand emulsions stabilised by polyelectrolyte complexes prepared from non-surface-active synthetic polyelectrolytes.

\section{EXPERIMENTAL}

\section{Materials}

Poly(4-styrene sulfonate) sodium salt, PSSNa, and poly(allylamine hydrochloride), PAH, were purchased from Alfa Aesar and were used as received. Their structures and the average molecular weight $\left(\mathrm{M}_{\mathrm{w}}\right)$ given by the supplier are shown in Table 1 . The $\mathrm{M}_{\mathrm{w}}$ of PSSNa is around half that of PAH. The oil used for preparing emulsions was $n$-dodecane (Alfa Aesar, > 99\%). It was passed twice through a basic alumina column (particle size: 0.063-0.200 mm, Merk $\mathrm{kGaA}$ ) to remove polar impurities. Water was first passed through a reverse osmosis unit and 
then a Milli-Q reagent water system (Millipore). After treatment, its surface tension measured with a Krüss $\mathrm{K} 11$ tensiometer and Wilhelmy plate was $72.0 \mathrm{mN} \mathrm{m}^{-1}$ at $25{ }^{\circ} \mathrm{C}$. Hydrochloric acid, $\mathrm{HCl}$ (37\%) and sodium hydroxide, $\mathrm{NaOH}$ (> 97\%) were purchased from Fisher Scientific and were used as received.

\section{Methods}

(a) Preparation and characterisation of aqueous PEC dispersions

A potentiometric titration for PAH was performed with a calibrated $\mathrm{pH}$ meter (3510, Jenway) to determine the degree of ionisation as a function of $\mathrm{pH}$. The natural $\mathrm{pH}$ of a $1 \mathrm{~g} \mathrm{~L}^{-1} \mathrm{PAH}$ solution was 4.2. The $\mathrm{pH}$ was decreased to 2.5 with $\mathrm{HCl}$ and the solution was titrated with 0.1 $\mathrm{M} \mathrm{NaOH}$. Individual PEL solutions of different concentrations (from 0.1 to $20 \mathrm{~g} \mathrm{~L}^{-1}$ ) were prepared by weighing the corresponding amount of each PEL and dissolving it in Milli-Q water. PEL solutions were adjusted to the desired $\mathrm{pH}$ with $\mathrm{NaOH}$ and $\mathrm{HCl}$ solutions of various concentrations. Aqueous PEC dispersions of different mole fractions of PSSNa (XPSSNa) were obtained by mixing known volumes of each individual PEL of a fixed concentration and $\mathrm{pH}$ with a magnetic stirrer (VWR VMS-C7, stirrer speed = 3) at room temperature. Here, x refers to the mole fraction calculated with the values of $\mathrm{M}_{\mathrm{w}}$ given in Table 1. We quote the initial $\mathrm{pH}$ before mixing in all cases. All solutions were prepared in $14 \mathrm{~mL}$ screw-cap glass vials under a specific mixing procedure to obtain reproducible results. The protocol detailed in the previous papers was followed. ${ }^{37,38}$ The PAH solution was added sequentially with a micropipette up to the total desired volume into the PSSNa solution. This was done to allow the added polyelectrolyte to interact with the oppositely charged species present in the vial. Therefore, as an example, for the dispersion with XPSSNa $=0.83$, each PAH addition was of $375 \mu \mathrm{L}$. With this protocol, the total mixing time for all the samples was $3 \mathrm{~min}$. After the complete addition of the PAH solution, mixing was kept at the same speed for an additional minute.

For aqueous PEC dispersions prepared at low polyelectrolyte concentration $\left(0.1 \mathrm{~g} \mathrm{~L}^{-1}\right)$, dynamic light scattering (DLS) employing the cumulant method ${ }^{39}$ was used to determine the average diameter of the complex present. The study was carried out at different XPSSNa and $\mathrm{pH}$ in the absence of added electrolyte. Measurements were performed at $25{ }^{\circ} \mathrm{C}$ using a Zetasizer Nanoseries NanoZS (ZEN3600, Malvern Instruments) and samples were placed in a plastic disposal cuvette of $1 \mathrm{~cm}$ path length. The instrument was equipped with a $4 \mathrm{~mW}$ He-Ne laser beam as a light source, operating at $\lambda=633 \mathrm{~nm}$ under a scattering angle of $173^{\circ}$. The results are given as the average of three measurements. The zeta potential was measured at $25^{\circ} \mathrm{C}$ by 
the same instrument. Measurements were made by introducing a universal dip cell (ZEN1002, Malvern Instruments) inside a plastic disposal cuvette. Henry's equation and the Smoluchowski approximation ${ }^{40}$ were used to convert measured electrophoretic mobilities to zeta potentials. Each value was averaged from three parallel measurements. The refractive index of water was obtained using a refractometer (M46 313, Hilger) and a sodium lamp ( $\lambda=$ $589 \mathrm{~nm})$ at $25{ }^{\circ} \mathrm{C}$ and was 1.333 . The intensity-weighted mean diameter derived from the cumulant analysis is reported, for which the dispersant refractive index and its viscosity are required.

For aqueous PEC dispersions prepared at higher [PEL], the type of associative phase separation (precipitation or complex coacervation) across the XPSSNa and $\mathrm{pH}$ range was assessed by visual inspection and optical microscope images. A drop of the aqueous PEC dispersion was placed on a glass slide (Fisher Scientific) and micrographs were taken using an Olympus BX-51 microscope fitted with a DP70 digital camera. The size distribution of the entities obtained at high [PEL] at different $\mathrm{pH}$ was measured with a Malvern Mastersizer 2000 (Malvern Instruments) fitted with a small volume sample dispersion unit. About $1250 \mu \mathrm{L}$ of the dispersion were diluted in $100 \mathrm{~mL}$ of Milli-Q water at a specific $\mathrm{pH}$ in the dispersion unit, stirred at 2,000 rpm. Each value was averaged from three parallel measurements. The particle diameter reported in the case of a monomodal distribution was the mass median diameter $d(0.5)$. The width of the distribution was given by the span value, calculated as shown in equation 1. For multimodal distributions, the size at the peak maximum is reported.

$$
\operatorname{span}=\frac{d(0.9)-d(0.1)}{d(0.5)}
$$

where $d(0.9)$ is the particle diameter for which $90 \%$ of the sample is below this size and $d(0.1)$ is the particle diameter for which $10 \%$ of the sample is below this size. The stability of the dispersions after two months was assessed visually.

The amount of unreacted PAH or PSSNa in selected aqueous PEC dispersions around charge neutrality prepared from $5 \mathrm{~g} \mathrm{~L}^{-1}$ PEL solutions at unmodified $\mathrm{pH}$ was assessed. In order to do so, aqueous PEC dispersions at different $\mathrm{X}_{\mathrm{PSSNa}}(0.68,0.83$ and 0.95$)$ were prepared and centrifuged with a minicentrifuge (Minispin plus, Eppendorf) at 10,000 rpm for $10 \mathrm{~min}$ to separate the PEC particles from the supernatant. Afterwards, different volumes $(0.5,1$ and 2 $\mathrm{mL})$ of PSSNa and PAH solutions of different concentrations $\left(0.1,1\right.$ and $\left.5 \mathrm{~g} \mathrm{~L}^{-1}\right)$ were added to $5 \mathrm{~mL}$ of the supernatant. The formation of PEC after the addition of PAH or PSSNa indicated 
the presence in the supernatant of free PSSNa or PAH, respectively. The amount of noncomplexed PAH or PSSNa in the supernatant was quantified through fluorescence measurements with a LS55 Fluorescence spectrometer (Perkin Elmer) in a four clear windows quartz cuvette taking advantage of the fact that each polyelectrolyte is fluorescent on its own. The slit width was set to $10 \mathrm{~nm}$ in all cases. Standard PSSNa $(0.01,0.025,0.05,0.075$ and 0.1 $\left.\mathrm{g} \mathrm{L}^{-1}\right)$ and PAH $\left(0.5,1.25,2.5,3.75\right.$ and $\left.5 \mathrm{~g} \mathrm{~L}^{-1}\right)$ solutions of different concentrations were prepared and the emission spectra was obtained after exciting the solutions at $\lambda=300 \mathrm{~nm}$. A calibration curve for each polyelectrolyte was built by plotting the maximum intensity $(\lambda=382$ $\mathrm{nm}$ for PSSNa and $\lambda=405 \mathrm{~nm}$ for PAH) versus PEL concentration. The intensity of emission of the supernatant prepared at different XPSSNa $(0.68,0.83$ and 0.95$)$ was recorded and interpolated in the correspondent calibration curve, which allowed the amount of free polyelectrolyte to be quantified. The percentage of free PEL was calculated as the ratio of the concentration of free PEL after complexation to the initial PEL concentration.

(b) Preparation and characterisation of emulsions

Emulsions composed of an aqueous PEC dispersion and $n$-dodecane were prepared in $14 \mathrm{~mL}$ screw-cap glass vials. The two phases were emulsified with an Ultra-Turrax homogenizer (IKA ${ }^{\circledR}$ T25 digital) with a dispersing head of $8 \mathrm{~mm}$ (stator diameter). Mixing was maintained for $2 \mathrm{~min}$ at a constant speed of 13,200 rpm. Different sets of emulsions were systematically prepared by varying one of the following parameters each time: concentration of the starting PEL solutions, XPSSNa, $\mathrm{pH}$ of the initial PEL solutions and oil volume fraction. Immediately after preparation, the emulsion type was inferred from the drop test. Photos and optical microscope images of emulsions were taken after preparation and after two months to assess the emulsion stability. Micrographs were obtained on a dimple glass slide (Fisher Scientific) with a cover slip (Scientific Laboratory Supplies LTD) using an Olympus BX-51 microscope fitted with a DP70 digital camera. The mean droplet diameter of the emulsion was calculated from at least fifty individual droplets on digital micrographs with Image $1.47 \mathrm{v}$ software. The amount of oil released from the emulsion after two months was measured by weight, after removing it carefully from above the emulsion with a Pasteur pipette. The amount of aqueous phase resolved after two months was assessed by measuring its height. Therefore, the fraction of oil $\left(f_{o}\right)$ or water $\left(f_{w}\right)$ released is calculated as the amount of oil or water resolved after two months relative to the initial amount of oil or water used in the preparation of the emulsion. 
Selected emulsions with different oil volume fractions were imaged with cryo-scanning electron microscopy (cryo-SEM). A small volume of emulsion was mounted on an aluminium sample holder (diameter $\sim 10 \mathrm{~mm}$ ) with a spatula. The sample was plunged into liquid nitrogen, previously turned into a slush to decrease the temperature to $-210^{\circ} \mathrm{C}$. The frozen sample was placed inside the cryo-preparation chamber (PP3010T, Quorum Technologies Ltd.) where it was fractured with a sharp knife at $-140{ }^{\circ} \mathrm{C}$ under high vacuum. Sublimation of the surface water (ice) was performed inside the Zeiss EVO 60 SEM chamber at $-75{ }^{\circ} \mathrm{C}$ for 10 min to obtain a clearer image of the droplet interface. Afterwards, the sample was coated with platinum to a thickness of $\sim 2 \mathrm{~nm}$ in the preparation chamber. Finally, it was transferred back to the SEM chamber for imaging at a voltage of $15 \mathrm{kV}$ and a probe current of $30 \mathrm{pA}$.

Confocal micrographs of aqueous PEC dispersions and emulsions were taken with a confocal laser scanning microscope (LSM 710, Carl Zeiss, Germany) equipped with a $30 \mathrm{~mW}$ Diode 405 laser. Samples were placed in a dimple glass slide with a cover slip. The data was acquired with EC Plan-Neofluar 10x/0.30 and 20x/0.5 and Plan-Apocromat 63x/1.40 oil DIC M27 objectives. For selected emulsions, scans were performed along the Z-axis and a 3D image was built with the ZEN 2010 software (Carl Zeiss).

The viscosity of emulsions prepared at different oil volume fractions was measured at $25{ }^{\circ} \mathrm{C}$ with a Bohlin CV 120 rheometer (Bohlin Instruments) using a $20 \mathrm{~mm}$ diameter parallel plate geometry. Temperature control was done with a Peltier plate. The gap between the two plates was set to $500 \mu \mathrm{m}$ for all emulsions. Measurements were done at controlled stress (from 0.18 to $100 \mathrm{~Pa}$ ). Freshly prepared emulsions were carefully placed on the lower plate of the rheometer. The upper plate was slowly lowered onto the sample until the pre-set gap size was reached. Any excess of emulsion was gently removed with a tissue.

\section{RESULTS AND DISCUSSION}

\section{(A) Characterisation of aqueous PEC dispersions}

The polyelectrolyte system reported here consists of a mixture of PSSNa and PAH. PSSNa is a strong polyacid and therefore fully ionised at all $\mathrm{pH}$. However, $\mathrm{PAH}$ is a weak polybase and consequently its degree of ionisation varies with $\mathrm{pH}$. A potentiometric titration of a $1 \mathrm{~g} \mathrm{~L}^{-1}$ PAH solution was carried out against $\mathrm{NaOH}$ as described in the experimental section. From that, the curve of the degree of ionisation as a function of $\mathrm{pH}$ and the $\mathrm{p} K_{\mathrm{a}}$ were obtained (Figure 
$\mathrm{S} 1)$. The $\mathrm{p} K_{\mathrm{a}}$ value for our sample was found to be 8.1, consistent with literature values ranging from 8 to $9^{41,42}$ but lower than the typical $\mathrm{p} K_{\mathrm{a}}$ of a low molecular weight aliphatic amine which is around 10.7..$^{43}$ The behaviour of weak polyelectrolytes upon increasing or decreasing the charge density is not comparable to that of simple electrolytes. For PAH, due to the electrostatic repulsion between positively charged groups within the polymer chain, as the fraction of protonated amine groups increases it becomes progressively more difficult to protonate.

(i) Low $[$ PEL]

As mentioned above, the charge of PAH varies with $\mathrm{pH}$. Consequently, its interaction with PSSNa to form the so-called polyelectrolyte complexes will be significantly affected. This was already posed for the system comprising a strong polybase (PDADMAC) and a weak polyacid (PAANa) after a systematic and comprehensive study over a wide $\mathrm{pH}$ range (2 to 10). ${ }^{38}$ Therefore, for the analysis of this system at low PEL concentration, three different $\mathrm{pH}$ were selected to cover the entire $\mathrm{pH}$ range. These were $\mathrm{pH}=2$, unmodified $\mathrm{pH}$ and $\mathrm{pH}=10$. The ionisation of $\mathrm{PAH}$ is considered to be $100 \%$ and $25 \%$ for $\mathrm{pH}=2$ and 10 , respectively. The $\mathrm{pH}$ of a $0.1 \mathrm{~g} \mathrm{~L}^{-1} \mathrm{PAH}$ solution at unmodified $\mathrm{pH}$ is 4.80 , which relates to a degree of ionisation of 93\%. Across all pH, PSSNa is fully ionised (100\%).

Aqueous PEC dispersions were first characterised in terms of their average diameter and zeta potential. The appearance of aqueous PEC dispersions prepared from $0.1 \mathrm{~g} \mathrm{~L}^{-1}$ PEL solutions at different XPSSNa and $\mathrm{pH}$ is shown in Figure S2(a). In general, all aqueous PEC dispersions and solutions of the neat polymers are transparent and colourless. However, at $\mathrm{pH}=10$ a few dispersions appear bluish (XPSNa $=0.34$ and 0.47 ) or show signs of precipitation ( $\mathrm{XPSSNa}_{\mathrm{PSN}}=0.67$ ). The $\mathrm{pH}$ of the resulting aqueous $\mathrm{PEC}$ dispersions was measured after preparation. For $\mathrm{pH}=2$ and 10 the $\mathrm{pH}$ did not vary considerably compared to that of the individual PEL solutions. In contrast, for aqueous PEC dispersions prepared at unmodified $\mathrm{pH}$, the $\mathrm{pH}$ varied from 5.09 to 5.84 due to the difference in $\mathrm{pH}$ of each PEL solution ( $\mathrm{pH}=5.68$ for $0.1 \mathrm{~g} \mathrm{~L}^{-1}$ PSSNa solution). However, for sake of simplicity, a $\mathrm{pH} \sim 5.5$ and consequently a degree of ionisation of PAH of $\sim 85 \%$ was assigned to all aqueous PEC dispersions at unmodified $\mathrm{pH}$.

The average diameter and zeta potential of the entities present in the above dispersions was measured and are shown in Figure 1 at different pH. For the three pH (Figure 1(a)-(c)), a plot of the average diameter versus XPSSNa displays a maximum of a few hundred nm. At unmodified $\mathrm{pH}(\sim 5.5)$, the result for XPSSNa $=0.83$ was not included as the value obtained from the three repetitions was not reproducible despite being higher than the rest $(\sim 450 \mathrm{~nm})$. After carefully 
examining the literature dealing with different polyelectrolyte combinations, we conclude that there is not full agreement about the dependence of the particle size on the mixing ratio. In some cases, ${ }^{44-46}$ the plot displays a minimum around the point of charge neutralization, while other authors ${ }^{47,48}$ report that the largest size is achieved when all the charges are compensated.

The plot of the zeta potential against XPSSNa displays a sigmoidal shape changing from positive to negative values for the three $\mathrm{pH}$, Figure 1(d). The sign reversal occurs at a relatively high XPSSNa and is linked to the maximum displayed in the average diameter. This could be explained by the difference in the molecular weight of the two polyelectrolytes (Table 1). The $\mathrm{M}_{\mathrm{w}}$ of PSSNa is around half of that of PAH so a higher amount of sulfonate groups must be available to achieve charge neutralisation. This is in line with ref. 37 . Bago Rodriguez et al. ${ }^{37}$ measured the zeta potential for the system containing PDADMAC (weak) and PSSNa (strong) assessing the effect of the $\mathrm{M}_{\mathrm{w}}$ of PSSNa at constant $\mathrm{M}_{\mathrm{w}}$ of PDADMAC. The XPSSNa at zeta potential sign reversal occurred at a lower value when the difference in the $M_{w}$ of the two polyelectrolytes was high. Moreover, for the system shown here it is worth noting that the change in the sign of the zeta potential shifts to slightly lower $\mathrm{X}_{\mathrm{PSSNa}}$ by increasing the $\mathrm{pH}$, as expected. At $\mathrm{pH}=10$, the degree of ionisation of $\mathrm{PAH}$ is $25 \%$, therefore a higher amount of protonated amine groups is needed to overcome all the negative charges of PSSNa compared to the situation at $\mathrm{pH}=2$ where both polyelectrolytes are fully ionised. This has been proved by plotting the zeta potential against $N_{P S S N a}$ in Figure S2(b). $N_{P S S N a}$ is defined by:

$$
N_{P S S N a}=\frac{n_{P S S N a}}{n_{P S S N a}+n_{P A H}}
$$

where $n_{P S S N a}$ and $n_{P A H}$ are the number of moles of PSSNa and PAH, respectively, calculated using the molar mass per unit charge (given in Table 1) and the degree of ionisation of PAH at each pH. Unlike Figure 1(d), $N_{P S S N a}$ at sign reversal occurs around 0.9 for the three $\mathrm{pH}$. This also supports the findings for the mixture of PDADMAC and PAANa, where the change in the sign of the zeta potential occurred at lower XPAANa as $\mathrm{pH}$ increased. ${ }^{38}$

(ii) High [PEL]

(a) Effect of $X_{P S S N a}$ at selected $p H$

The effect of $\mathrm{pH}$ and XPSSNa was investigated at high PEL concentrations. After increasing [PEL] to $10 \mathrm{~g} \mathrm{~L}^{-1}$, the appearance of aqueous PEC dispersions at $\mathrm{pH}=2$, unmodified and 10 changed significantly compared to the one at low [PEL] as seen in Figure 2. At $\mathrm{pH}=2$ and unmodified $\mathrm{pH}$ (pH of PSSNa and PAH solution are 4.49 and 3.59, respectively), the appearance is similar 
so only that for unmodified $\mathrm{pH}$ is shown (Figure 2(a)). After preparation, dispersions appear turbid, with that prepared at XPSSNa $=0.83$ being the most turbid. This implies a higher volume fraction of PEC compared to the other XPSSNa. Moreover, this mole fraction corresponds to charge neutralisation as probed previously using zeta potential measurements. Optical micrographs were taken from all the dispersions and solid particles with irregular shape were observed. An example is shown in Figure 2(c, left) for XPsSNa $=0.48$. After two months, particles sediment, being the highest sediment obtained at XPSSNa $=0.83$ (as expected). At these pH (2 and unmodified), both PAH and PSSNa are fully ionised. Therefore, a strong electrostatic interaction is expected to occur which is entirely consistent with the formation of a solid-liquid type of phase separation (precipitate).

The appearance of aqueous PEC dispersions prepared at $\mathrm{pH}=10$ differs significantly to the previous studied $\mathrm{pH}$, Figure 2(b). A close inspection after preparation using optical microscopy reveals two different types of associative phase separation (precipitation and complex coacervation). At $\mathrm{X}_{\mathrm{PSSNa}}<0.83$, both precipitates and coacervate droplets were detected. Moreover, at specific XPSSNa (0.35 and 0.48) PEC particles are highly aggregated and remain glued at the bottom of the vial and around the walls. An example showing the coexistence of both solid particles and coacervate droplets is shown in Figure 2(c, right). Precipitates have irregular shape while coacervate droplets are spherical and liquid in nature. Co-existence of both coacervate droplets and solid particles has been reported previously. ${ }^{38,49}$ At XPSSNa $=0.83$ and 0.95 however, only coacervate droplets were detected. At $\mathrm{pH}=10$, the degree of ionisation of PSSNa and PAH is $100 \%$ and $25 \%$, respectively. Therefore, a weak electrostatic interaction occurs, in line with the formation of coacervate droplets.

An interesting feature to highlight for the dispersions at $\mathrm{pH}=10$ is the remarkable change in the stability of PEC coacervates with time observed at specific mole fractions. For instance, at $\mathrm{XPSSNa}=0.68$, despite being the most turbid initially, only after four hours (not shown) it turned completely transparent. This is explained by the coalescence of coacervate droplets forming the so-called coacervate phase (a viscous and concentrated polymer phase) that settled at the bottom of the vial. This $\mathrm{XPSSNa}_{\mathrm{PS}}$ is close to charge neutralisation from zeta potential measurements. Moreover, it is worth noting the enhanced stability against coalescence of coacervate droplets in aqueous PEC dispersions prepared at XPSSNa $=0.83$ and 0.95 . The appearance of the dispersions after three months is still turbid although a clear liquid depleted in coacervate droplets appears on top. Despite the coacervate droplets settling down gently, 
they do not coalesce with each other as they easily re-disperse recovering the appearance of the initial dispersion. Probably, at XPSSNa $=0.68$, coacervate droplets are uncharged which enables their coalescence. However, at higher XPSSNa, the surface of coacervate droplets might be negatively charged due to excess PSSNa and this could prevent their coalescence due to electrostatic repulsion. This distinct behaviour regarding the stability of coacervate droplets was already highlighted in our previous publication for the system PDADMAC+PAANa. ${ }^{38}$ At $\mathrm{pH}=6$ coalescence of coacervate droplets occurred with time while from $\mathrm{pH}=7$ to 10 coacervate droplets were stable to coalescence. Despite several experiments carried out to elucidate the internal structure and composition at the interface of coacervate droplets, ${ }^{38}$ these points still remain unresolved. The same study as described above was carried out at two lower polyelectrolyte concentrations ( 1 and $5 \mathrm{~g} \mathrm{~L}^{-1}$ in Figures S3 and S4, respectively) and the same pattern of behaviour was observed.

As seen from Figures 2, S3 and S4, the amount of PEC particles is maximum at $\mathrm{X}_{\mathrm{PSSNa}}=0.83$ (around charge neutrality) and decreases drastically around this value. The amount of unreacted $\mathrm{PAH}$ or PSSNa in the supernatant of selected aqueous PEC dispersions around charge neutrality prepared from $5 \mathrm{~g} \mathrm{~L}^{-1}$ PEL solutions was identified and quantified to obtain information about the yield of PEC formation at different XPSSNa. The studied XPSSNa were 0.68, 0.83 and 0.95 . Three different volumes $(0.5,1$ and $2 \mathrm{~mL})$ of PSSNa and PAH solutions of different concentrations $\left(0.1,1\right.$ and $\left.5 \mathrm{~g} \mathrm{~L}^{-1}\right)$ were added to the supernatants. For XPSSNa $=0.68$, free PSSNa does not seem to be present in the supernatant as it remained completely transparent after the addition of PAH solution (Figure S5(a)). On the contrary, unreacted PAH appears to be in the supernatant as a high amount of PEC particles was formed after the addition of a $5 \mathrm{~g}$ $\mathrm{L}^{-1}$ PSSNa solution (Figure S5(b)). The amount of free PAH was quantified via fluorescence measurements by interpolation on the calibration curve the intensity of the sample at $\lambda_{\text {em }}=405$ $\mathrm{nm}$ (Figure S6). The amount of free PAH was found to be $90.4 \%$. For $\mathrm{XPSSNa}=0.83$, residual PSSNa is found in the supernatant as the dispersion turns slightly bluish after the addition of specific volumes and concentrations of PAH (Figure S7(a)). The amount of free PSSNa was estimated to be $0.4 \%$ from fluorescence measurements (Figure S8). Therefore, virtually almost all PSSNa is complexed with PAH. Finally, for $\mathrm{XPSSNa}_{\mathrm{PS}}=0.95$ unreacted PSSNa is detected in the supernatant, as after the addition of PAH high amounts of PEC particles are formed (Figure S9(a)). The amount of free PSSNa estimated from fluorescence measurements was found to be 66\% (Figure S9(c)). At high XPSSNa, there are not enough PAH chains available to interact with excess PSSNa and so more PSSNa chains appear free in the supernatant. 


\section{(b) Effect of $p H$ at fixed $x_{P S S N a}$}

The influence of $\mathrm{pH}$ on the formation of PEC was studied in more detail by working at a fixed PEL concentration of $5 \mathrm{~g} \mathrm{~L}^{-1}$ and XPSSNa $=0.83$. The appearance of dispersions prepared at six different $\mathrm{pH}$ one day and two months after preparation is shown in Figure 3. Three different patterns of behaviour can be distinguished. From pH 2 to 6, only solid particles were detected both visually and under an optical microscope. Their appearance is the same as that described previously for aqueous PEC dispersions prepared at $\mathrm{pH}=2$ and unmodified at different XPSSNa. As discussed, the degree of ionisation of $\mathrm{PAH}$ at these $\mathrm{pH}$ is $100 \%$. Consequently, a strong electrostatic interaction is expected to occur with the oppositely charged polyelectrolyte resulting in precipitate formation. At $\mathrm{pH}=8$ both precipitate and coacervate droplets were identified while at $\mathrm{pH}=10$ only coacervate droplets were formed. The degree of ionisation of $\mathrm{PAH}$ at these $\mathrm{pH}$ is lower than $100 \%$ and as discussed previously this weak electrostatic interaction could explain the formation of coacervate droplets. Finally, at $\mathrm{pH}=12$ a transparent solution is recovered. The degree of ionisation of $\mathrm{PAH}$ is $0 \%$ so no electrostatic interaction is expected to occur and individual polymer chains dissolve in solution.

The size distributions of PEC (particles and coacervate droplets) prepared at different pH were determined with a Mastersizer and Zetasizer. The average particle diameter for each $\mathrm{pH}$ is summarized in Table 2. From pH 2 to 6, the size distribution is relatively monomodal (span values $\sim 1.1$ ) and centred around $16 \mu \mathrm{m}$. Moreover, there is no significant effect of $\mathrm{pH}$. The distribution obtained for $\mathrm{pH}=2$ is shown in Figure S10(a) as an example. Interestingly, the average diameter measured for the PEC obtained at $\mathrm{pH}=2$ at low polyelectrolyte concentration was in the nanometre range, in contrast to the micrometre range reported here. By increasing the concentration of PEL, the number of complexes can increase at constant size, larger aggregates can form at fixed number or both scenarios can occur simultaneously. ${ }^{38}$ Starchenko et al. found that by increasing the PEL concentration for the system PDADMAC-PSSNa, the aggregation of primary particles is accelerated giving rise to an increase in the size of secondary particles. ${ }^{50}$ At $\mathrm{pH}=8$, two distributions were obtained, in agreement with the two types of associative phase separation identified through optical microscopy. The distribution centred at $202 \mathrm{~nm}$ is that of coacervate droplets whereas the second distribution (maximum at $11.2 \mu \mathrm{m}$ ) corresponds to solid particles (Figure S10(b)). At pH = 10 only one peak at $\sim 150 \mathrm{~nm}$ is displayed, attributed to coacervate droplets (Figure S10(c)), while at $\mathrm{pH}=12$ no scattering occurred as no complex is formed. The transition precipitate - precipitate and coacervate coacervate - polymer solution is thus observed by progressively reducing the charge on PAH 
(increasing the $\mathrm{pH}$ ). This is in agreement with what is generally accepted: strong interactions between PEL lead to precipitates while coacervates arise from weak interactions. However, for the system PDADMAC-PAANa, this behaviour was not followed precisely as coacervate droplets were formed at high $\mathrm{pH}$ when both polyelectrolytes were expected to be fully ionised. ${ }^{38}$

Regarding the long term stability of aqueous PEC dispersions (Figure 3(b)), from pH 2 to 6 particles sedimented and the height of the sediment is similar. At $\mathrm{pH}=8$ particles sediment but the amount of particles is lower compared to acidic $\mathrm{pH}$. Dispersions at $\mathrm{pH}=8$ and 10 remain bluish despite a clear liquid depleted in coacervate droplets appearing on top. Therefore, at these two $\mathrm{pH}$, coacervate droplets stable to coalescence are formed in line with the results shown previously. At the selected XPSSNa (0.83), the charge of the aggregates is expected to change with $\mathrm{pH}$. Therefore, zeta potential measurements were carried out from these dispersions but prepared at a lower [PEL] of $0.1 \mathrm{~g} \mathrm{~L}^{-1}$. The zeta potential changes from positive to negative by increasing the $\mathrm{pH}$ as expected (Figure S11). At low $\mathrm{pH}$, when both PEL are fully ionised, an excess of positive charge is present. The zeta potential then decreases slightly until it becomes negative by $\mathrm{pH}=8$. From this point onwards, the excess charge is negative as $\mathrm{PAH}$ becomes less charged.

\section{(B) Stabilisation of oil-in-water emulsions}

After identifying the different types of phase separation across the XPSSNa and $\mathrm{pH}$ ranges, the ability to prepare emulsions with these dispersions and $n$-dodecane was assessed. The parameters evaluated are XPSSNa, PEL concentration and $\mathrm{pH}$ as well as the oil volume fraction. From these results, together with the ones obtained from previous systematic investigations of other polyelectrolyte combinations, we put forward the conditions to obtain the most stable emulsions with PEC as emulsifier.

\section{(a) Effect of XPSSNa}

Emulsions were prepared with $n$-dodecane $\left(\phi_{o}=0.2\right)$ and aqueous PEC dispersions at different XPSSNa from $10 \mathrm{~g} \mathrm{~L}^{-1}$ PEL solutions at unmodified $\mathrm{pH}$ as well as with individual PEL solutions (Figure 4). All emulsions were oil-in-water as inferred from the drop test after preparation. Regarding the emulsion stability of the individual PEL, PAH (XPSNa $=0$ ) is not surface-active as the emulsion completely phase separates after homogenisation. On the contrary, despite PSSNa (XPSSNa $=1)$ showing some surface activity initially, after two months the amount of oil 
coalesced is $46 \%$. Emulsions stable to different extents were however prepared from aqueous PEC dispersions. The most stable emulsions were obtained around charge neutrality (XPSSNa $0.8)$. At these mole fractions ( 0.79 and 0.83 ) all the particles migrate to the emulsion cream as the resolved aqueous phase becomes completely transparent with no evidence of complexes. Moreover, and more crucial for the stability study, the fraction of cream generated is higher and the average droplet diameter is the lowest and remains unaltered after two months compared to the other XPSSNa (Figure 4(c)). The oil resolved after two months was measured by weight for all emulsions. The amount of oil released for emulsions prepared close to charge neutrality, $\mathrm{XPSSNa}_{\mathrm{P}}=0.79$ and 0.83 , was $3.8 \%$ and $1.1 \%$, respectively. For the other XPSSNa, this value was substantially higher and varied from $4 \%$ to $12 \%$, although the emulsion with a XPSSNa $=0.48$ displayed a relatively higher value (37\%). Therefore, PEC enable long term emulsion stability compared to the individual PEL. As concluded from the results in Figure 2(a), around charge neutralisation a higher amount of neutral PEC particles are formed, which are more suitable for emulsion stabilisation compared to the individual hydrophilic polyelectrolytes. This is in agreement with a previous publication on emulsions prepared with a different PEL combination (PDADMAC + PSSNa). ${ }^{37}$

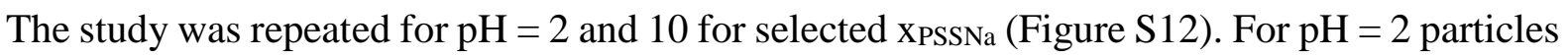
were obtained across the mole fraction range and the results are similar to the ones obtained at unmodified $\mathrm{pH}$, as expected. The most stable emulsion with the smallest average droplet diameter $(29 \pm 8 \mu \mathrm{m})$, the highest fraction of cream and the lowest amount of oil coalesced after two months ( $3 \%$ ) was obtained at a XPSSNa $=0.83$. However, at $\mathrm{pH}=10$ either coacervate droplets or coexistence of both particles and coacervate droplets were present across the mole fraction range. Consequently, the emulsion behaviour was different to that obtained for the other $\mathrm{pH}$. The majority of emulsions completely coalesce and only the ones obtained close to the XPSSNa extremes are stable in the long term (Figure S12(c)). The ability of coacervate droplets to stabilise emulsions was assessed in ref. 38. However, the experimental conditions in that case had to be optimised in order to obtain stable emulsions. Many parameters such as the viscosity or the spreading of one phase over another have to be considered which makes this system difficult to fully understand. ${ }^{38}$ In conclusion, the stability of emulsions prepared with PEC particles ( $\mathrm{pH}=2$ and unmodified) is higher than that from dispersions containing coacervate droplets $(\mathrm{pH}=10)$. 
(b) Effect of [PEL]

In particle-stabilised emulsions, particle concentration plays an important role in emulsion stabilisation. ${ }^{51}$ At low stabiliser concentration (emulsifier-poor régime) droplets are partially covered so they coalesce to a limited extent. By increasing the particle concentration, the degree of surface coverage increases and this results in the decrease of the mean droplet size. Finally, at high emulsifier concentration (emulsifier-rich régime), the average drop size remains unaltered. ${ }^{51-53}$ Aqueous PEC dispersions (XPSSNa $=0.83$ ) were prepared from PEL solutions of different concentrations at unmodified $\mathrm{pH}$. Afterwards, emulsions were prepared with $n$-dodecane $\left(\phi_{o}=0.2\right.$ ) in order to evaluate the influence of PEL concentration on emulsion stability. The appearance of emulsions once creaming had ended is shown in Figure 5(a). Two different behaviours can be distinguished. Between 1 and $10 \mathrm{~g} \mathrm{~L}^{-1} \mathrm{PEL}$, all the particles migrate to the cream, the fraction of cream increases slightly (Figure 5(b)) and the average droplet diameter decreases first and then remains unaltered (Figure 5(c)). Above $10 \mathrm{~g} \mathrm{~L}^{-1} \mathrm{PEL}$, not all the particles migrate to the cream, the average droplet diameter increases and the fraction of cream is reduced slightly. The amount of oil coalesced after two months is less than $2 \%$ for emulsions prepared at a [PEL] between 1 and $10 \mathrm{~g} \mathrm{~L}^{-1}$ and slightly higher $(<8 \%)$ for emulsions prepared at higher [PEL] (Figure 5(b)).

Selected optical microscope images of emulsions prepared at different [PEL] are shown in Figure S13. For the first [PEL] range, the system behaves as described by the limited coalescence model, i.e. the average droplet diameter decreases with polyelectrolyte concentration until it reaches a point where emulsion drops are fully covered by particles so the excess particles form a network in the continuous phase and the droplet diameter does not decrease further. Some examples of emulsions stabilised by various kinds of particles following this pattern of behaviour are encountered in the literature. ${ }^{21,37,51-53}$ However, for emulsions prepared at polyelectrolyte concentrations higher than $10 \mathrm{~g} \mathrm{~L}^{-1}$, the behaviour differs from the one expected above. We have to be aware, however, that here particles are formed from the interaction of two oppositely charged polyelectrolytes, unlike the case of traditional solid particles. Therefore, at high polyelectrolyte concentration, entanglements of polymer chains are expected to be greater and this can induce further aggregation of the complexes.

The average diameter of the aqueous complexes prepared at selected [PEL] were measured with the Mastersizer. In Figure S14(a), particle size distributions are shown for PEC particles in aqueous PEC dispersions prepared at four [PEL]. The values of $\mathrm{d}(0.1), \mathrm{d}(0.5)$ and $\mathrm{d}(0.9)$ 
together with the span value are plotted in Figure S14(b). PEC particles obtained at [PEL] = 1 and $5 \mathrm{~g} \mathrm{~L}^{-1}$ are relatively monodisperse in size. However, as soon as the [PEL] increases, the distribution gets wider and larger particles are present. Larger particles are dislodged easily from the oil-water interface of emulsion drops. This would explain the decrease in emulsion stability at $[\mathrm{PEL}]>10 \mathrm{~g} \mathrm{~L}^{-1}$.

(c) Effect of $\mathrm{pH}$

Emulsions were prepared with $n$-dodecane $\left(\phi_{o}=0.2\right)$ and aqueous PEC dispersions shown in Figure 3 for $[\mathrm{PEL}]=5 \mathrm{~g} \mathrm{~L}^{-1}$ and $\mathrm{XPSSNa}_{\mathrm{P}}=0.83$ (Figure 6). For emulsions prepared at $\mathrm{pH}=2$ to 6, all the particles migrate to the cream (Figure 6(b)), the average droplet diameter is about 30 $\mu \mathrm{m}$ (Figure 6(c)) and the amount of oil coalesced after two months is $<1.5 \%$. Moreover, the average droplet diameter remains unaltered with time. When coacervate droplets are present in dispersions ( $\mathrm{pH}=8$ and 10), the average droplet diameter increases, the cream height decreases and the amount of oil released also increases ( $2 \%$ and $5 \%$, respectively). The average droplet diameter changes more with time and droplets are deformed at $\mathrm{pH}=10$ compared to those obtained at other $\mathrm{pH}$ (Figure S15(a)). Finally, at $\mathrm{pH}=12$, the average droplet diameter decreases to the same level as the one reported for low $\mathrm{pH}$. In this case, however, the stabilisation is given by free polymer chains of PAH as it is not expected to be ionized. In order to check this assumption, emulsions were prepared from $5 \mathrm{~g} \mathrm{~L}^{-1} \mathrm{PEL}$ solutions (Figure S15(b)). During homogenisation, both emulsions generated high amount of foam which completely collapsed a few minutes after preparation. $\mathrm{PAH}$ ( $0 \%$ ionised) is surface-active and the emulsion remains stable for at least two months with a relatively low amount of oil coalesced (9\%). The same behaviour was noted for a $5 \mathrm{~g} \mathrm{~L}^{-1}$ PAANa solution at $\mathrm{pH}=2$ ( $0 \%$ ionised). ${ }^{38}$ On the contrary, for PSSNa (100\% ionised), despite being surface-active initially the amount of oil coalesced after two months is $40 \%$. Therefore, to sum up, emulsion stabilisation by PEC particles is more successful than for PEC coacervates. Non-ionized polyelectrolytes behave as polymers and hence can stabilise emulsions.

(d) Effect of oil volume fraction

Some emulsions stabilised by solid particles undergo catastrophic phase inversion by varying the oil:water ratio. ${ }^{54,55}$ In order to evaluate the influence of this parameter in our system, emulsions with $n$-dodecane at different $\phi_{o}$ were prepared with an aqueous PEC particle dispersion $\left(\mathrm{XPSSNa}=0.83,[\mathrm{PEL}]=10 \mathrm{~g} \mathrm{~L}^{-1}\right.$ and $\mathrm{pH}=2$ ). The particle concentration with respect to the emulsion thus varies. The appearance of emulsions at different $\phi_{0}$ once creaming is over 
is shown in Figure 7(a). Selected optical micrographs are shown in Figure 7(c). The average droplet diameter increases with $\phi_{0}$ as shown in Figure 8(a) due to the progressive decrease in the particle concentration in the system. At high $\phi_{o}$ droplets are deformed and the viscosity of emulsions increases considerably. This indicates the formation of high internal phase emulsions (HIPEs). ${ }^{56,57}$ For monodisperse spheres arranged in face centered cubic crystalline structure, the maximum close packing fraction that can be accomplished is $0.74 .{ }^{57}$ However, for emulsions, this value can be exceeded and fractions of disperse phase up to 0.95 have been reported. ${ }^{58}$ Spherical liquid droplets, unlike solid spheres, can be compressed into polyhedra separated by thin films of continuous phase. ${ }^{56}$ For our system, HIPEs were obtained up to $\phi_{o}=$ 0.85 (Figure 7(a)) as no emulsion could be prepared at $\phi_{o}=0.9$. The formation of HIPEs is not common in particle-stabilised systems although several examples are found in the literature. ${ }^{52,59-62}$ It is difficult to predict which type of emulsifier will render HIPEs. The film of emulsifier must be rigid enough to resist breaking but flexible enough to adjust to changing prevailing conditions. ${ }^{57}$ Ikem et al. reported the stabilisation of Pickering o/w HIPEs up to $\phi_{o}$ $=0.92$ using silica nanoparticles hydrophobized by oleic acid. ${ }^{61}$

The appearance of the emulsions remains almost unaltered after two months (Figure 7(b)). The average droplet diameter does not significantly change at low $\phi_{o}$, whereas at $\phi_{o}=0.8$ and 0.85 it increases substantially. Figure 8(b) shows the fraction of oil and water released after two months. The amount of oil released is $<6 \%$ apart from the emulsion prepared at $\phi_{o}=0.8(16 \%)$. Moreover, creaming was fully inhibited at $\phi_{0} \geq 0.7$ as no aqueous phase was resolved. As a control, emulsions with individual PEL solutions at the same conditions ([PEL] $=10 \mathrm{~g} \mathrm{~L}^{-1}, \mathrm{pH}$ $=2$ ) were prepared and the long term stability was assessed. Plots of $f_{o}$ and $f_{w}$ are shown in Figure 9. It can be seen that PAH is not surface-active for most oil volume fractions $\left(f_{o}\right.$ and $f_{w}$ close to $100 \%)$. However, although PSSNa is surface-active initially, values of $f_{o}$ and $f_{w}$ after 2 months are substantially higher than for emulsions prepared from aqueous PEC dispersions (Figure 8(b)). PEC particles thus enable long term emulsion stability and HIPEs can be formed at high $\phi_{0}$.

Cryo-SEM images of emulsions across the oil volume fraction range were obtained. At $\phi_{o}=$ 0.5 (Figure 10(a)), spherical and monodisperse entities of diameter around $110 \mathrm{~nm}$ are detected both at the interface (LHS) and in the aqueous continuous phase (RHS). Surprisingly, at $\phi_{o}=$ 0.6 (Figure 10(b)), elongated structures were detected (length $\sim 600 \mathrm{~nm}$, diameter $\sim 130 \mathrm{~nm}$ ). It is worth noting that the diameter of the rod-like shape entities is of the same order as that of the spherical entities. This suggests that the elongated structures are formed by merging of 
several spherical entities. Other images at $\phi_{o}=0.6$ are shown in Figure S16(a). For emulsions with $\phi_{o}=0.7$ (Figure S17) both spherical and rod-like entities were observed of the same dimensions as those presented earlier. At $\phi_{o}=0.8$ only spherical entities were detected (Figure S16(b). Moreover, the surface is not fully covered as in the previous cases due to a lower amount of particles present in the emulsion. The reason for the change in the morphology of the PEC entities in emulsions is unclear as the aqueous PEC dispersion used to prepare the emulsions is the same for all $\phi_{0}$. However, PEC particles can be considered to be soft particles as they form through electrostatic interaction between two charged polymers. Due to their soft nature, PEC particles could hold oil in their structures or the application of shear during emulsification might be responsible. Moreover, a discrepancy in the size of the PEC particles can be gleaned by comparing the cryo-SEM images with optical microscope images (Figure 2(c)) and the results from size measurements of aqueous PEC dispersions (Table 2). Nanometer-sized particles are identified from cryo-SEM images of emulsions while particles in the micrometer range are measured with the Mastersizer and optical microscopy images of aqueous dispersions. Since PEC particles are aggregated structures, it is likely that the high shear used during homogenisation could result in their size reduction.

Taking advantage of the fact that each PEL is fluorescent after being excited at $405 \mathrm{~nm}$ (Figure S18(a)), confocal micrographs of emulsions prepared with PEC particles were taken in order to visualise the location of particles after emulsification. Optical images and the corresponding confocal micrographs of an aqueous PEC dispersion (XPSSNa $=0.83)$ and emulsions prepared at different $\phi_{o}$ are shown in Figure S18(b) and Figure 11(a-c), respectively. PEC particles in water (shown in red) are fluorescent on their own after being excited at $405 \mathrm{~nm}$. Regarding emulsions, at $\phi_{o}=0.2$, particles are found at the interface of oil drops and excess particles form a network in the continuous aqueous phase (Figure 11(a)). At higher $\phi_{o}$ (Figure 11(b and c)), particles are only detected at the oil-water interface. A 3D image of the emulsion drops prepared at $\phi_{o}=0.8$ (Figure 11(d)) was obtained by taking a series of confocal micrographs at different sample depths (Figure S19). Particles are only visible at the oil-water interface and oil droplets appear to be partially covered, in agreement with cryo-SEM images at this $\phi_{0}$. At high $\phi_{0}$, the long term mechanical stability of emulsions and their gel-like rheology is thought to arise from the occurrence of particle bridging. ${ }^{63-65}$ In relation to this, stable emulsions have been prepared even if droplets are sparsely covered. ${ }^{66,67}$ In this case, particles accumulate spontaneously at the point of contact between two droplets preventing coalescence. ${ }^{65}$ The occurrence of particle bridging in this system at $\phi_{o}=0.8$ was checked using the highest magnification available in 
CLSM. Lee et al. used the same technique to report particle bridging in emulsions stabilised by fluorescent silica microspheres. ${ }^{68}$ Unfortunately, as shown in Figure S20, individual PEC particles cannot be detected in the thin film between two drops (due to their small diameter) as a red halo is observed instead.

In order to prove the increase in viscosity upon increasing $\phi_{0}$, rheology measurements were carried out for the above emulsions. Curves of viscosity against shear stress are shown in Figure 12(a). The viscosity of emulsions with $\phi_{o}=0.8$ and 0.85 could not be measured because the gap size was close to the average droplet diameter. By plotting the viscosity at a specific shear stress (10 Pa) against $\phi_{o}$, (Figure 12(b)) it can be seen that the viscosity increases markedly from $\phi_{o}=0.1$ to 0.5 and then decreases for $\phi_{o}=0.6$ and 0.7 . On the one hand, the concentration of particles with respect to the emulsion decreases with $\phi_{o}$. It is known that the particle concentration influences the viscosity of a solution. ${ }^{69}$ On the other hand, the average droplet diameter increases with $\phi_{0}$. Evidence exists that rheological properties are strongly influenced by the droplet size. ${ }^{70,71}$ Pal showed that fine emulsions have much higher viscosities than the corresponding coarse emulsions at the same $\phi_{o} .{ }^{71}$ Also, the viscosity of an emulsion increases with $\phi_{o}$ at constant droplet diameter. ${ }^{72}$ Therefore this effect, together with the decrease in particle concentration, could result in the viscosity decreasing from its expected value at high volume fractions of oil.

\section{CONCLUSIONS}

In the present paper, the characterisation of aqueous PEC dispersions and emulsions was carried out for the PEC system between a weak cationic (PAH) and a strong anionic (PSSNa) polyelectrolyte. For aqueous PEC dispersions, the transition precipitate precipitate/coacervate - coacervate - polymer solution occurs by increasing the $\mathrm{pH}$. At low $\mathrm{pH}$, both PEL are fully ionised and therefore precipitates are formed as a result of strong electrostatic interactions. By increasing the $\mathrm{pH}$, the degree of ionisation of $\mathrm{PAH}$ decreases and weak electrostatic interactions ensue, which supports the formation of coacervate droplets.

For the emulsion study, oil-in-water emulsions were prepared in all cases. The most stable emulsions with PEC particles are obtained around charge neutralisation. As neither PAH nor PSSNa are surface-active in the long term it can be claimed that emulsion stability is attributable to PEC particles. Emulsion stabilisation decreases considerably when coacervate 
droplets are present in the dispersion. High internal phase emulsions, identified by the occurrence of deformed droplets and gel-like emulsions with high viscosities, are obtained at high oil volume fractions with PEC particles, which is unusual in particle-stabilised systems. CLSM was used to visualise PEC particles by taking advantage of the inherent fluorescence of the PEL. At low $\phi_{0}$, both the aqueous continuous phase and the oil-water interface were fully covered by PEC particles. Conversely, at high $\phi_{o}$, particles were placed only at the interface of dispersed droplets, which were sparsely covered.

\section{ACKNOWLEDGEMENTS}

We are grateful to Shiseido (Japan) for a fully funded PhD studentship to AMBR and to Mr. A. Sinclair (University of Hull) for the SEM images. 


\section{REFERENCES}

[1] Binks, B. P. Emulsions - Recent Advances and Understanding. In Modern Aspects of Emulsion Science; Binks, B. P., Ed.; Royal Society of Chemistry: Cambridge, 1998; pp 1-48.

[2] Kargar, M.; Fayazmanesh, K.; Alavi, M.; Spyropoulos, F.; Norton, I. T. Investigation into the potential ability of Pickering emulsions (food-grade particles) to enhance the oxidative stability of oil-in-water emulsions. J. Colloid Interface Sci. 2012, 366, 209-215.

[3] Simovic, S.; Ghouchi-Eskandar, N.; Prestidge, C. A. Pickering emulsions for dermal delivery. J. Drug Delivery Sci. Technol. 2011, 21, 123-133.

[4] Frelichowska, J.; Bolzinger, M.-A.; Pelletier, J.; Valour, J.-P.; Chevalier, Y. Topical delivery of lipophilic drugs from o/w Pickering emulsions. Int. J. Pharm. 2009, 371, 56-63.

[5] Pickering, S. U. Emulsions. J. Chem. Soc. Trans. 1907, 91, 2001-2021.

[6] Ramsden, W. Separation of solids in the surface-layers of solutions and suspensions (observations on surface-membranes, bubbles, emulsions, and mechanical coagulation). Preliminary account. Proc. Roy. Soc. 1903, 72, 156-164.

[7] Finkle, P.; Draper, H. D.; Hildebrand, J. H. The theory of emulsification. J. Am. Chem. Soc. 1923, 45, 2780-2788.

[8] Schulman, J. H.; Leja, J. Control of contact angles at the oil-water-solid interfaces. Emulsions stabilized by solid particles $\left(\mathrm{BaSO}_{4}\right)$. Trans. Faraday Soc. 1954, 50, 598-605.

[9] Abend, S.; Bonnke, N.; Gutschner, U.; Lagaly, G. Stabilization of emulsions by heterocoagulation of clay minerals and layered double hydroxides. Colloid Polym. Sci. 1998, 276, 730-737.

[10] Binks, B. P.; Liu, W.; Rodrigues, J. A. Novel Stabilization of Emulsions via the Heteroaggregation of Nanoparticles. Langmuir 2008, 24, 4443-4446.

[11] Nallamilli, T.; Mani, E.; Basavaraj, M. G. A Model for the Prediction of Droplet Size in Pickering Emulsions Stabilized by Oppositely Charged Particles. Langmuir 2014, 30, 9336-9345.

[12] Nallamilli, T.; Binks, B. P.; Mani, E.; Basavaraj, M. G. Stabilization of Pickering Emulsions with Oppositely Charged Latex Particles: Influence of Various Parameters and Particle Arrangement around Droplets. Langmuir 2015, 31, 11200-11208.

[13] Yan, N.; Gray, M. R.; Masliyah, J. H. On water-in-oil emulsions stabilized by fine solids. Colloids Surf. A 2001, 193, 97-107.

[14] Zhou, J.; Qiao, X.; Binks, B. P.; Sun, K.; Bai, M.; Li, Y.; Liu, Y. Magnetic Pickering emulsions stabilized by $\mathrm{Fe}_{3} \mathrm{O}_{4}$ nanoparticles. Langmuir 2011, 27, 3308-3316.

[15] Kim, J.; Cote, L. J.; Kim, F.; Yuan, W.; Shull, K. R.; Huang, J. Graphene oxide sheets at interfaces. J. Am. Chem. Soc. 2010, 132, 8180-8186.

[16] Binks, B. P.; Clint, J. H.; Whitby, C. P. Rheological behavior of water-in-oil emulsions stabilized by hydrophobic bentonite particles. Langmuir 2005, 21, 5307-5316.

[17] Wang, W.; Milani, A. H.; Cui, Z.; Zhu, M.; Saunders, B. R. Pickering emulsions stabilized by $\mathrm{pH}$-responsive microgels and their scalable transformation to robust submicrometer colloidoisomes with selective permeability. Langmuir 2017, 33, 8192-8200.

[18] Brugger, B.; Richtering W. Emulsions stabilized by stimuli-sensitive $\operatorname{poly}(N-$ isopropylacrylamide)-co-methacrylic acid polymers: microgels versus low molecular weight polymers. Langmuir 2008, 24, 7769-7777.

[19] Brugger, B.; Rosen, B. A.; Richtering, W. Microgels as stimuli-responsive stabilizers for emulsions. Langmuir 2008, 24, 12202-12208.

[20] Richtering, W. Responsive emulsions stabilized by stimuli-sensitive microgels: emulsions with special non-Pickering properties. Langmuir 2012, 28, 17218-17229. 
[21] Destribats, M.; Rouvet, M.; Gehin-Delval, C.; Schmitt, C.; Binks, B. P. Emulsions stabilized by whey protein microgel particles: towards food-grade Pickering emulsions. Soft Matter 2014, 10, 6941-6954.

[22] Destribats, M.; Lapeyre, V.; Sellier, E.; Leal-Calderon, F.; Ravaine, V.; Schmitt, V. Origin and control of adhesion between emulsion drops stabilized by thermally sensitive soft colloidal particles. Langmuir 2012, 28, 3744-3755.

[23] Style, R. W.; Isa, L.; Dufresne, E. R. Adsorption of soft particles at fluid interfaces. Soft Matter 2015, 11, 7412-7419.

[24] Albertsson, P. A.; Partition of cell particles and macromolecules: distribution and fractionation of cells, mitochondria, chlorophasts, viruses, proteins, nucleic acids, and antigen-antibody complexes in aqueous polymer two-phase systems, Wiley-Interscience: New York, 1971; pp 18-21.

[25] M.W. Beijerinck, Zbl. Bakt.,1896, 2, 627, 698.

[26] M.W. Beijerinck, Kolloid-Z., 1910, 7, 16.

[27] Olabis, O.; Robeson, L. M.; Shaw, M. T. Polymer-polymer miscibility; Academic Press: New York, 1979; p 2.

[28] Dobry, A.; Boyer-Kawenoki, F. Phase separation in polymer solution. J. Polym. Sci. 1947, 2, 90-100.

[29] Dautzenberg, H.; Philipp, B. Polyelectrolytes: Formation, Application and Characterisation; Hanser Publishers: New York, 1994; pp 1-45.

[30] Bungenberg de Jong, H. D.; Kruyt, H. R. Proc. Koninkl. Med. Akad. Wetershap. 1929, 32, 849-856.

[31] Comert, F.; Malanowski, A. J.; Azarikia, F.; Dubin, P. L. Coacervation and precipitation in polysaccharide-protein systems. Soft Matter 2016, 12, 4154-4161.

[32] Liu, X.; Haddou, M.; Grillo, I.; Mana, Z.; Chapel, J.-P.; Schatz, C. Early stage kinetics of polyelectrolyte complex coacervation monitored through stopped-flow light scattering. Soft Matter 2016, 12, 9030-9038.

[33] Evans, M.; Ratcliffe, I.; Williams, P. A. Emulsion stabilisation using polysaccharideprotein complexes. Curr. Opin. Colloid Interface Sci. 2013, 18, 272-282.

[34] Jourdain, L.; Leser, M. E.; Schmitt, C.; Michel, M.; Dickinson, E. Stability of emulsions containing sodium caseinate and dextran sulfate: relationship to complexation in solution. Food Hydrocolloids 2008, 22, 647-659.

[35] Stone, A. K.; Nickerson, M. T. Formation and functionality of whey protein isolate (kappa-, iota-, and lambda-type) carrageenan electrostatic complexes. Food Hydrocolloids 2012, 27, 271-277.

[36] Zinoviadou, K. G.; Scholten, E.; Moschakis, T.; Biliaderis, C. G. Properties of emulsions stabilised by sodium caseinate-chitosan complexes. Int. Dairy J. 2012, 26, 94-101.

[37] Bago Rodriguez, A. M.; Binks, B. P.; Sekine, T. Novel stabilization of emulsions by soft particles: polyelectrolyte complexes. Faraday Discuss. 2016, 191, 255-285.

[38] Bago Rodriguez, A. M.; Binks, B. P.; Sekine, T. Emulsion stabilization by complexes of oppositely charged synthetic polyelectrolytes. Soft Matter 2018, 14, 239-254.

[39] Koppel, D. E. Analysis of macromolecular polydispersity in intensity correlation spectroscopy: the method of cumulants. J. Chem. Phys. 1972, 57, 4814-4820.

[40] Smoluchowski, M. Handbuch der Electrizität und des Magnetismus; Barth: Leipzig, 1921; Vol. 2, p 366.

[41] Fang, M.; Kim, C. H.; Saupe, G. B.; Kim, H.; Waraksa, C. C.; Miwa, T.; Fujishima, A.; Mallouk, T. E. Layer-by-layer growth and condensation reactions of niobate and titanoniobate thin films. Chem. Mater. 1999, 11, 1526-1532.

[42] Choi, J.; Rubner, M. F. Influence of the Degree of Ionization on Weak Polyelectrolyte Multilayer Assembly. Macromolecules 2005, 38, 116-124. 
[43] Perrin, D. D.; Dempsey, B.; Sergeant, E. P. pKa Prediction for Organic Acids and Bases, Chapman and Hall: New York, 1981.

[44] Mende, M.; Buchhammer, H.-M.; Schwarz, S.; Petzold, G.; Jaeger, W. The Stability of Polyelectrolyte Complex Systems of Poly(diallydimethyl-ammonium chloride) with Different Polyanions. Macromol. Symp. 2004, 211, 121-133.

[45] Sotiropoulou, M.; Cincu, C.; Bokias, G.; Staikos, G. Water-soluble polyelectrolyte complexes formed by poly(diallyldimethylammonium chloride) and poly(sodium acrylate-cosodium 2-acrylamido-2-methyl-1-propanesulphonate)-graft-poly( $N, N$-dimethylacrylamide) copolymers. Polymer 2004, 45, 1563-1568.

[46] Matralis, A.; Sotiropoulou, M.; Bokias, G.; Staikos, G. Water-Soluble Stoichiometric Polyelectrolyte Complexes Based on Cationic Comb-Type Copolymers. Macromol. Chem. Phys. 2006, 207, 1018-1025.

[47] Gärdlund, L.; Wågberg L.; Norgren, M. New insights into the structure of polyelectrolyte complexes. J. Colloid Interface Sci. 2007, 312, 237-246.

[48] Mende, M.; Petzold, G.; Buchhammer, H. Polyelectrolyte complex formation between poly(diallyldimethyl-ammonium chloride) and copolymers of acrylamide and sodium-acrylate. Colloid Polym. Sci. 2002, 280, 342-351.

[49] Koetz, J.; Kosmella, S. Interactions between poly(diallyldimethylammonium chloride) and poly(acrylic acid) in dependence on polymer concentration, presented in part at the International Conference on Scaling Concepts and Complex Fluids, Italy, 1994.

[50] Starchenko, V.; Müller, M.; Lebovka, N. Sizing of PDADMAC/PSS complex aggregates by polyelectrolyte and salt concentration and PSS molecular weight. J. Phys. Chem. B 2012, 116, 14961-14967.

[51] Tcholakova, S.; Denkov, N. D.; Lips, A. Comparison of solid particles, globular proteins and surfactants as emulsifiers. Phys. Chem. Chem. Phys. 2008, 10, 1608-1627.

[52] Arditty, S.; Whitby, C. P.; Binks, B. P., Schmitt, V.; Leal-Calderon, F. Some general features of limited coalescence in solid-stabilized emulsions. Eur. Phys. J. E 2003, 11, 273281.

[53] Arditty, S.; Schmitt, V.; Giermanska-Kahn, J.; Leal-Calderon, F. Materials based on solidstabilized emulsions. J. Colloid Interface Sci. 2004, 275, 659-664.

[54] Binks, B. P.; Lumsdon, S. O. Catastrophic phase inversion of water-in-oil emulsions stabilised by hydrophobic silica. Langmuir 2000, 16, 2539-2547.

[55] Binks, B. P.; Rodrigues, J. A. Types of phase inversion of silica particle stabilized emulsions containing triglyceride oil. Langmuir 2003, 19, 4905-4912.

[56] Cameron, N. R.; Sherrington, D. C. High internal phase emulsions (HIPEs) - Structure, properties and use in polymer preparation. In Biopolymers liquid crystalline polymers phase emulsion; Advances in Polymer Science; Springer: Berlin, Heidelberg, 1996; Vol. 126.

[57] Lissant, K. J. Basic theory. In Emulsions and emulsion technology Part 1; Lissant, K. J., Ed.; Marcel Dekker Inc.: New York, 1974.

[58] Zhang, S.; Zhu, Y.; Hua, Y.; Jegat, C.; Chen, J.; Taha, M. Stability of surfactant-free high internal phase emulsions and its tailoring morphology of porous polymers based on the emulsions. Polymer 2011, 52, 4881-4890.

[59] Hu, Y.; Yin, S.; Zhu, J.; Qi, J.; Guo, J.; Wu, L.; Tang, C.; Yang, X. Fabrication and characterisation of novel Pickering emulsions and Pickering high internal emulsions stabilized by gliadin colloidal particles. Food Hydrocolloids 2016, 61, 300-310.

[60] Ikem, V. O.; Menner, A.; Bismarck, A. High-porosity macroporous polymers synthesized from titania-particle-stabilized medium and high internal phase emulsions. Langmuir 2010, 26, 8836-8841.

[61] Ikem, V. O.; Menner, A.; Bismark, A. High internal phase emulsions stabilised solely by functionalized silica particles. Angew. Chem. Int. Ed. 2008, 47, 8277-8279. 
[62] Capron, I.; Cathala, B. Surfactant-free high internal phase emulsions stabilized by cellulose nanocrystals. Biomacromolecules 2013, 14, 291-296.

[63] Thareja, P.; Velankar, S. Particle-induced bridging in immisible polymer blends. Rheol. Acta 2007, 46, 405-412.

[64] Thareja, P.; Velankar, S. Rheology of immiscible blends with particle-induced drop clusters. Rheol. Acta 2008, 47, 189-200.

[65] Horozov, T. S.; Binks, B. P. Particle-stabilized emulsions: A bilayer or a bridging monolayer? Angew. Chem. Int. Ed. 2006, 118, 787-790.

[66] Midmore, B. R. Preparation of a novel silica-stabilized oil/water emulsion. Colloids Surf. A 1998, 132, 257-265.

[67] Vignati, E.; Piazza, R. Pickering emulsions: interfacial tension, colloidal layer morphology and trapped-particle motion. Langmuir 2003, 19, 6650-6656.

[68] Lee, M. N.; Chan, H. K.; Mohraz, A. Characteristics of Pickering emulsion gels formed by droplet bridging. Langmuir 2012, 28, 3085-3091.

[69] Macosko, C. W. Rheology: principles, measurements and applications; Wiley-VCH: New York, 1994; pp 449.

[70] Malkin, A. Y.; Masalova, I.; Slatter, P.; Wilson, K. Effect of droplet size on the rheological properties of highly-concentrated w/o emulsions. Rheol. Acta 2004, 43, 584-591.

[71] Pal, R. Effect of droplet size on the rheology of emulsions. AIChE J. 1996, 42, 3181-3190.

[72] Pal, R. Shear viscosity behaviour of emulsions of two immiscible liquids. J. Colloid Interface Sci. 2000, 255, 359-366. 
Table 1. Characteristics of polyelectrolytes used.

\begin{tabular}{|c|c|c|c|c|c|}
\hline Polyelectrolyte & Repeat unit & $\mathrm{M}_{\mathrm{w}} / \mathrm{g} \mathrm{mol}^{-1}$ & $\begin{array}{c}\text { Molar mass } \\
\text { per unit } \\
\text { charge /g } \\
\text { mol }^{-1}\end{array}$ & Supplier & Appearance \\
\hline $\begin{array}{l}\text { Poly(allylamine } \\
\text { hydrochloride), } \\
\text { PAH }\end{array}$ & & $\begin{array}{l}120,000- \\
200,000\end{array}$ & 93.5 & $\begin{array}{l}\text { Alfa } \\
\text { Aesar® }\end{array}$ & $\begin{array}{l}\text { Yellow } \\
\text { powder }\end{array}$ \\
\hline $\begin{array}{l}\text { Poly(4- } \\
\text { styrenesulfonate), } \\
\text { PSSNa }\end{array}$ & & 75,000 & 206.2 & $\begin{array}{l}\text { Alfa } \\
\text { Aesar® }\end{array}$ & $\begin{array}{l}\text { Yellow } \\
\text { crystals }\end{array}$ \\
\hline
\end{tabular}


Table 2. Average diameter of PEC aggregates formed in $5 \mathrm{~g} \mathrm{~L}^{-1}$ aqueous PEL solutions (XPSSNa $=0.83$ ) at different $\mathrm{pH}$.

\begin{tabular}{|c|c|}
\hline $\mathbf{p H}$ & Average diameter \\
\hline 2 & $17.9 \pm 0.3 \mu \mathrm{m}$ \\
\hline 4 & $14.5 \pm 0.9 \mu \mathrm{m}$ \\
\hline 6 & $14.6 \pm 1.3 \mu \mathrm{m}$ \\
\hline 8 & $202 \pm 48 \mathrm{~nm} ; 11.2 \pm 0.6 \mu \mathrm{m}$ \\
\hline 10 & $148.7 \pm 6.1 \mathrm{~nm}$ \\
\hline 12 & - \\
\hline
\end{tabular}


Figure 1. Variation of (a-c) average particle diameter and (d) zeta potential with XPSSNa for aqueous PEC dispersions prepared from $0.1 \mathrm{~g} \mathrm{~L}^{-1}$ PEL solutions of PAH and PSSNa at different $\mathrm{pH}$ (given).
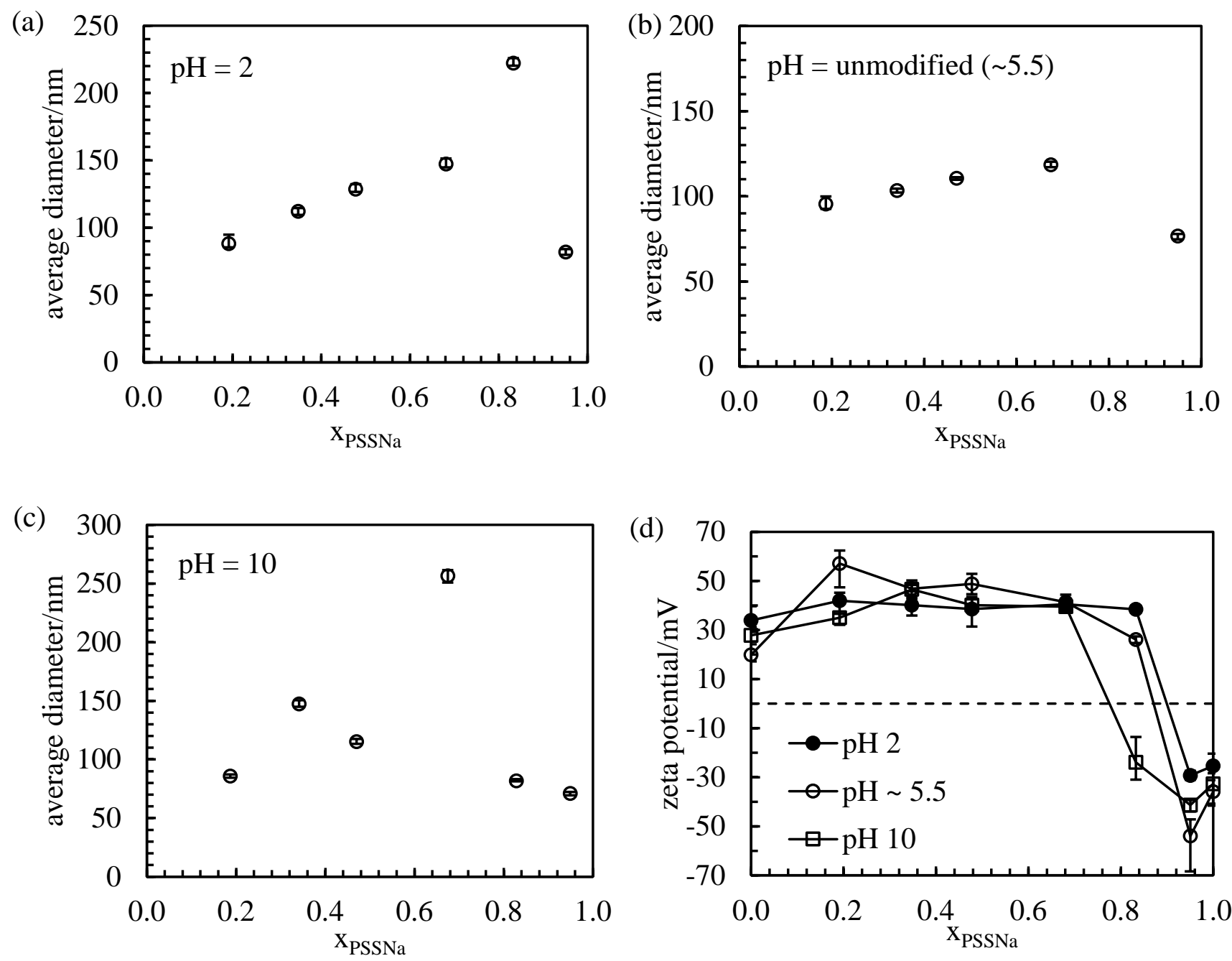
Figure 2. Appearance of aqueous PEC dispersions prepared from $10 \mathrm{~g} \mathrm{~L}^{-1} \mathrm{PEL}$ solutions at $\mathrm{pH}$ (a) unmodified ( 4) and (b) 10 at different XPSSNa (given) for two times. Scale bar $=1 \mathrm{~cm}$. (c) Optical microscope images of aqueous PEC dispersions ( $\left.\mathrm{XPSSNa}_{\mathrm{P}}=0.48\right)$ taken after preparation for unmodified $\mathrm{pH}$ (left) and $\mathrm{pH}=10$ (right).

(a)

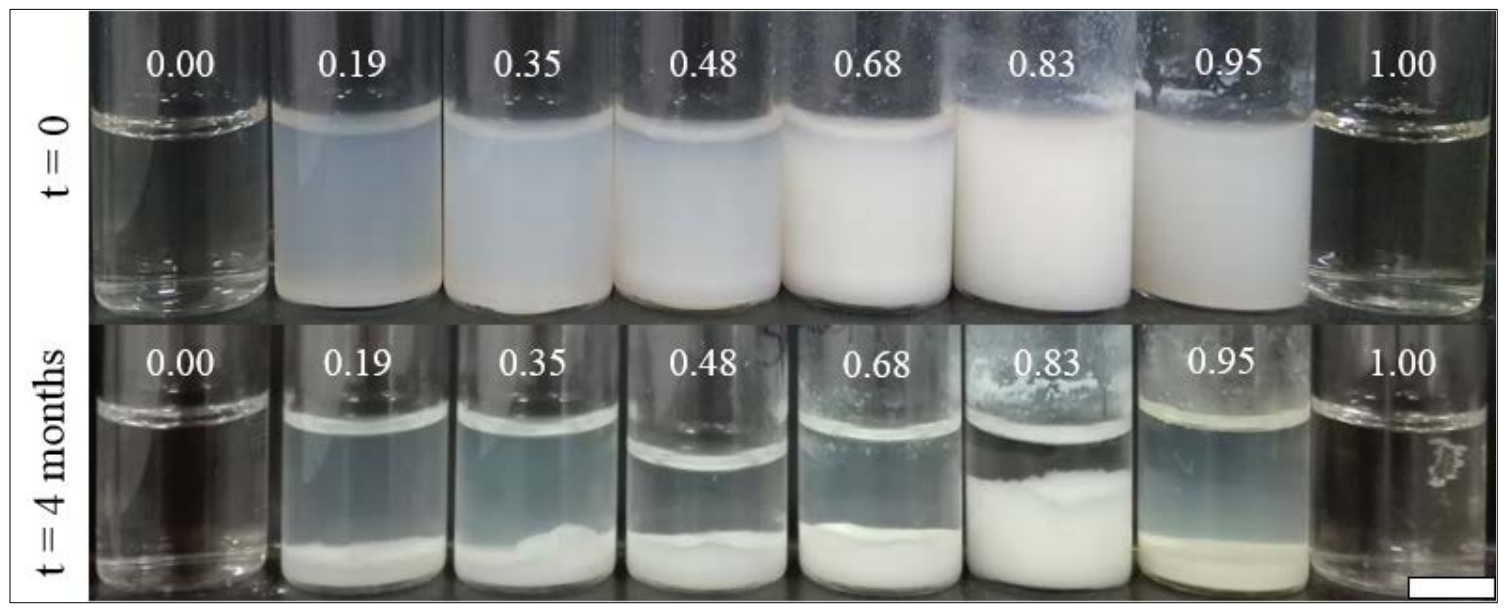

(b)

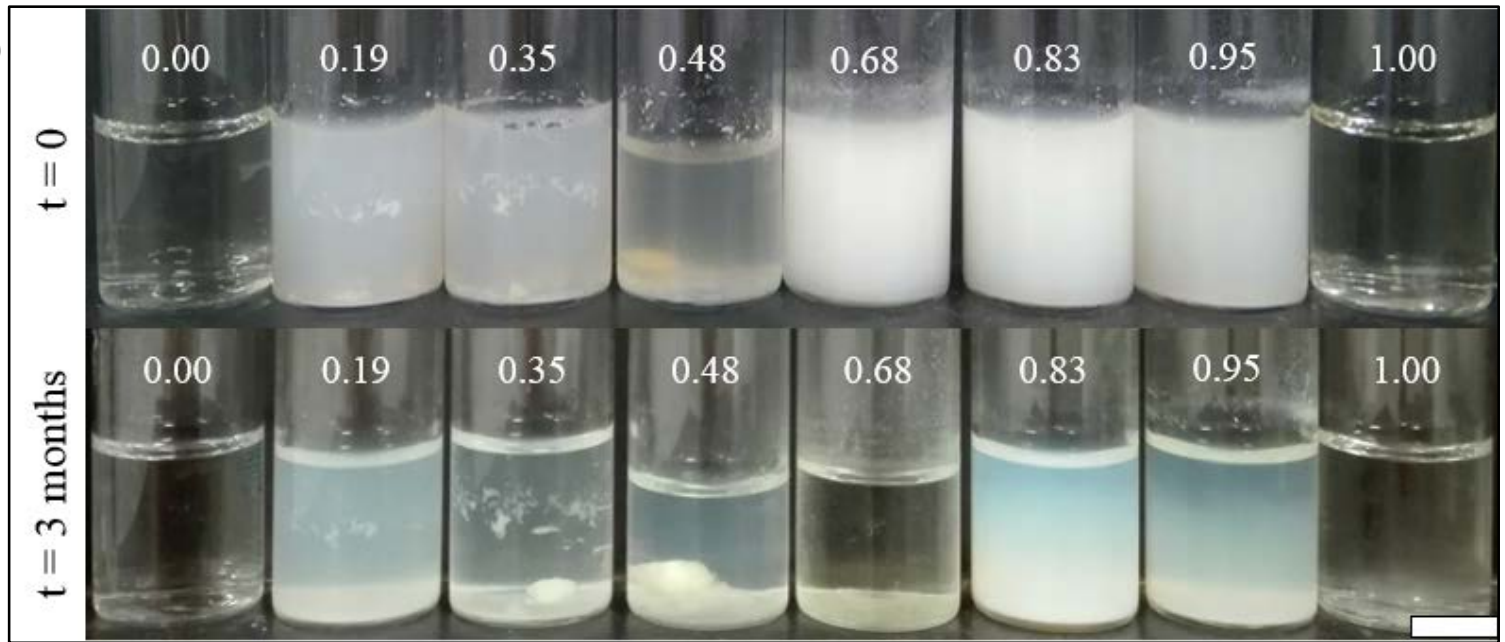

(c)

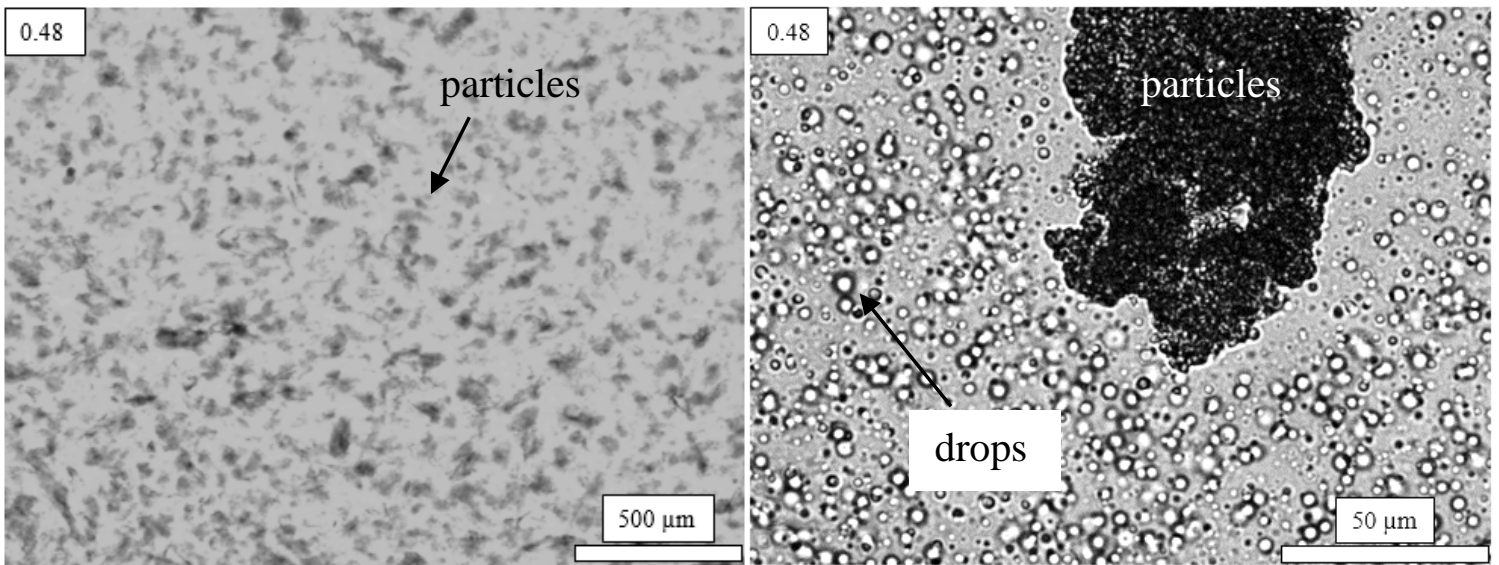


Figure 3. Appearance of aqueous PEC dispersions prepared from $5 \mathrm{~g} \mathrm{~L}^{-1} \mathrm{PEL}$ solutions (XPSSNa $=0.83$ ) at different $\mathrm{pH}$. Photos taken (a) 1 day and (b) 2 months after preparation. Scale bar $=$ $1 \mathrm{~cm}$.

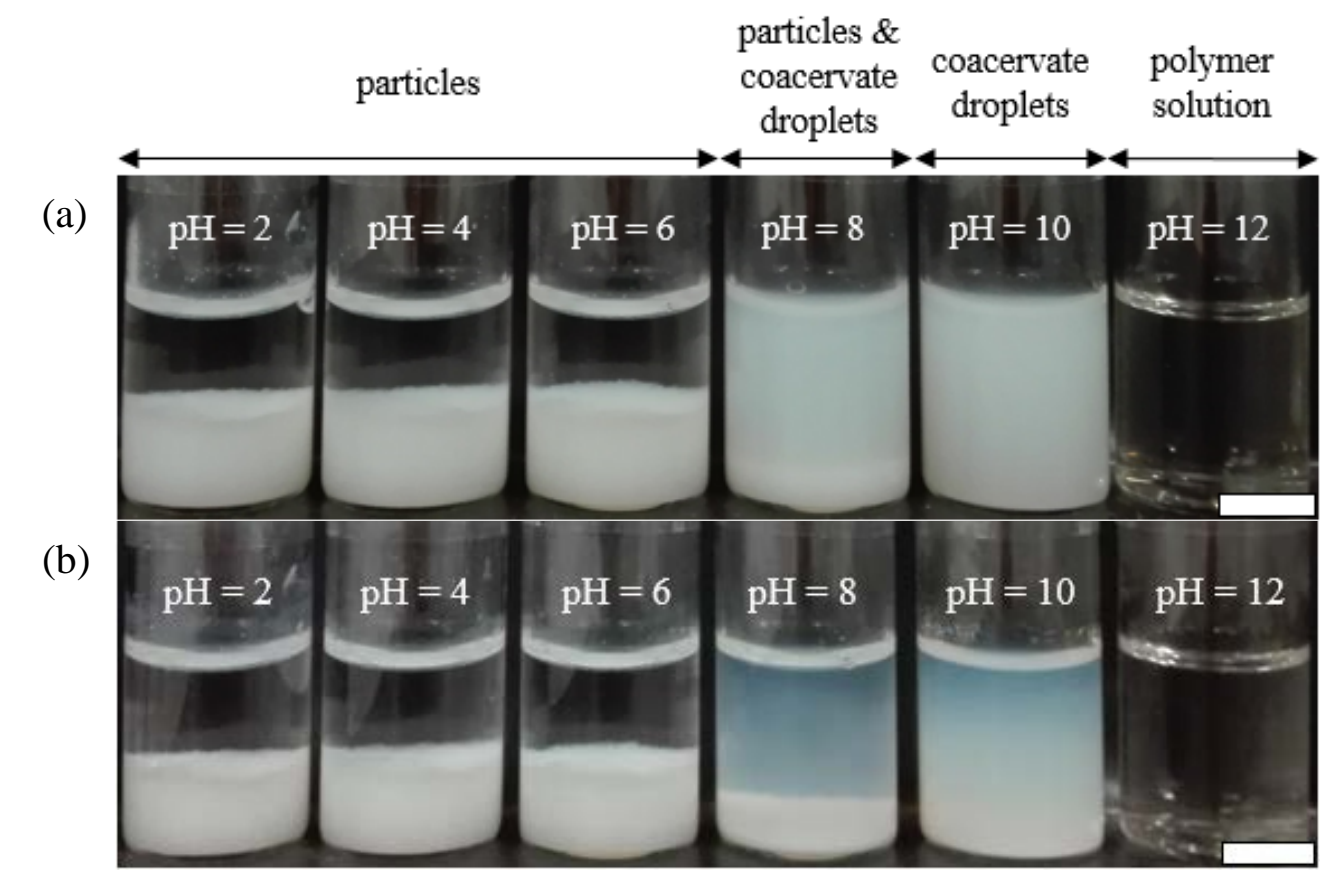


Figure 4. (a) Appearance of emulsions prepared with $n$-dodecane $\left(\phi_{o}=0.2\right)$ and aqueous PEC dispersions ([PEL] $=10 \mathrm{~g} \mathrm{~L}^{-1}, \mathrm{pH}=$ unmodified) at different XPSSNa given (a) $15 \mathrm{~min}$ and (b) 2 months after preparation. Scale bar $=1 \mathrm{~cm}$. (c) Plot of average droplet diameter versus $\mathrm{XPSSNa}_{\mathrm{P}}$ for the above emulsions.

(a)

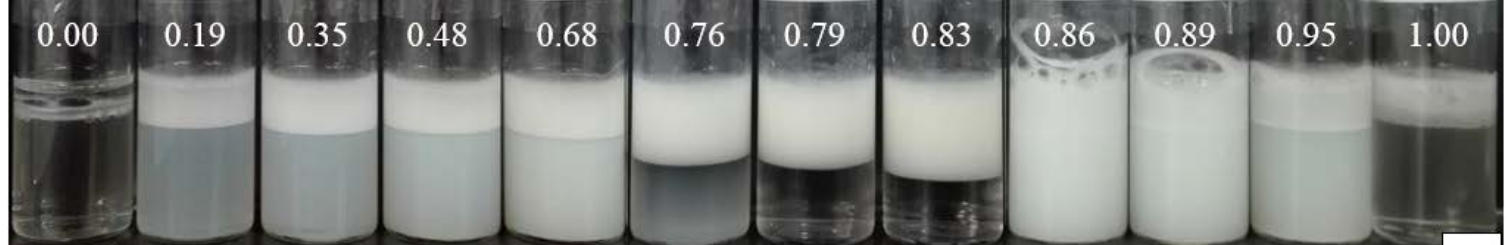

(b)

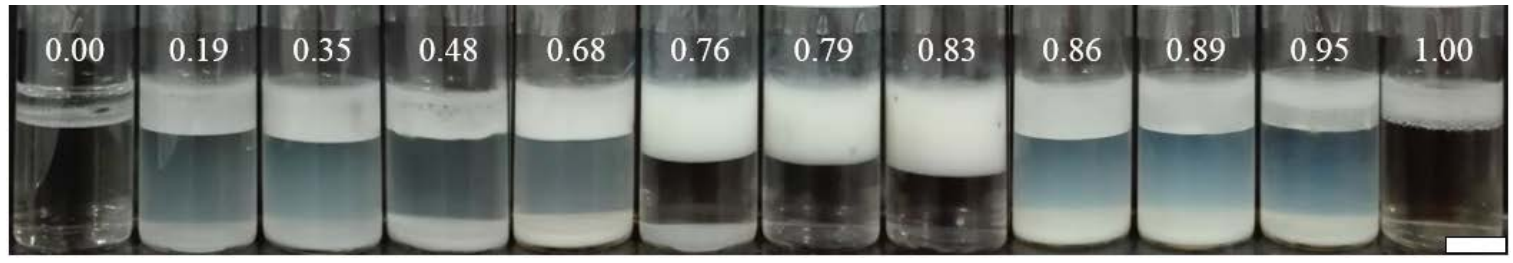

(c)

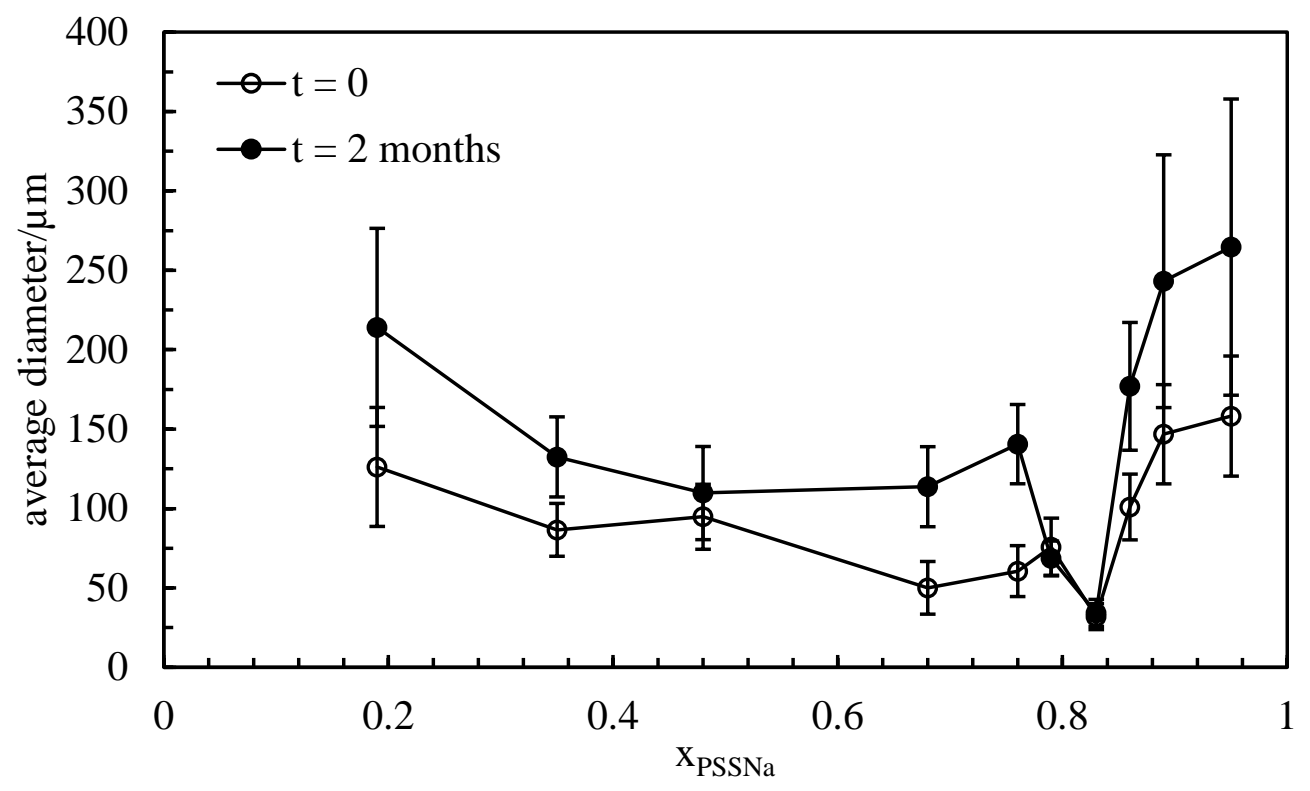


Figure 5. Appearance of emulsions prepared with $n$-dodecane $\left(\phi_{o}=0.2\right)$ and aqueous PEC dispersions (XPSSNa $=0.83, \mathrm{pH}=$ unmodified) at different [PEL] given in $\mathrm{g} \mathrm{L}^{-1}$ (a) $15 \mathrm{~min}$ and (b) 2 months after preparation. Scale bar $=1 \mathrm{~cm}$. (c) Plot of average droplet diameter and (d) fraction of cream two months after preparation versus [PEL] for the above emulsions.

(a)

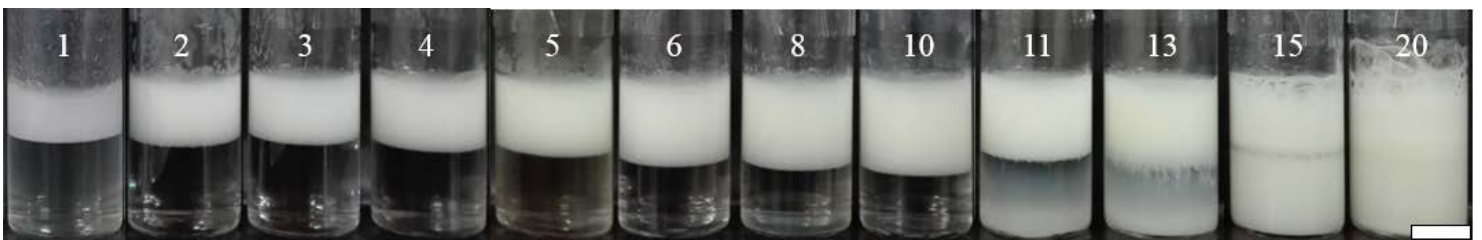

(b)

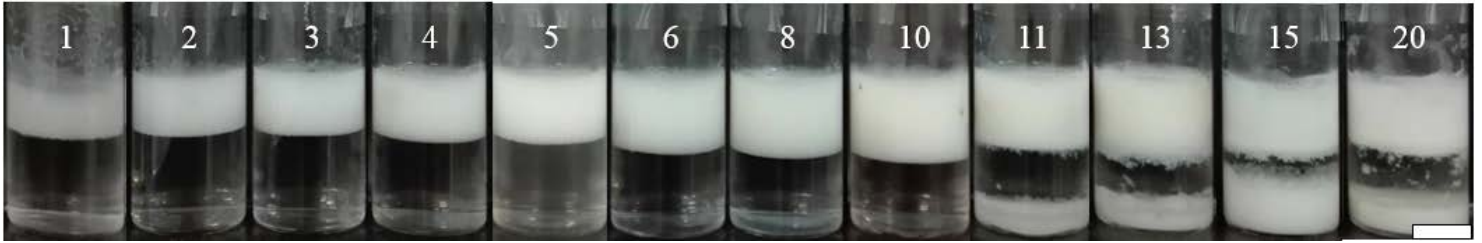

(c)

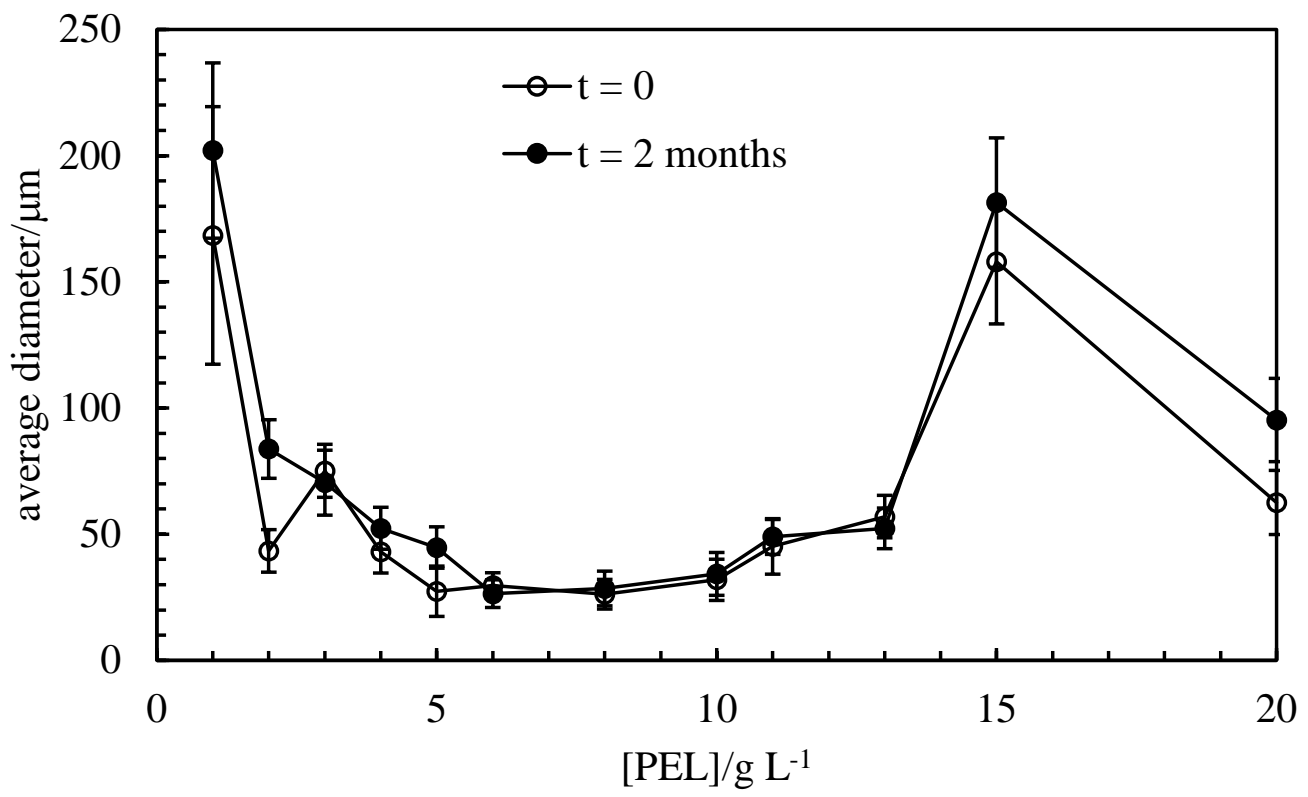

(d)

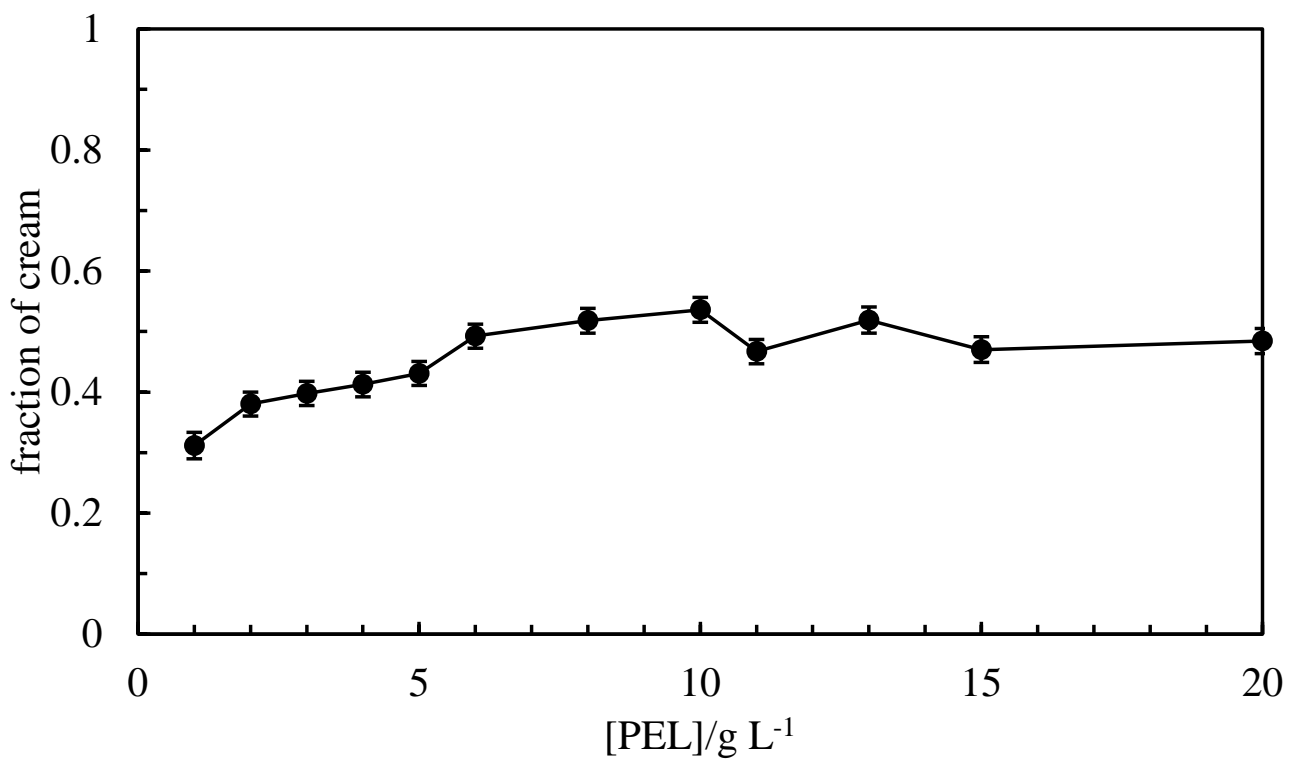


Figure 6. Appearance of emulsions prepared with $n$-dodecane $\left(\phi_{o}=0.20\right)$ and aqueous PEC dispersions (XPSSNa $=0.83,[\mathrm{PEL}]=5 \mathrm{~g} \mathrm{~L}^{-1}$ ) at different $\mathrm{pH}$ (given). Photos taken (a) 1 day and (b) 2 months after preparation. (c) Plot of average droplet diameter versus $\mathrm{pH}$ at two times.

(a)

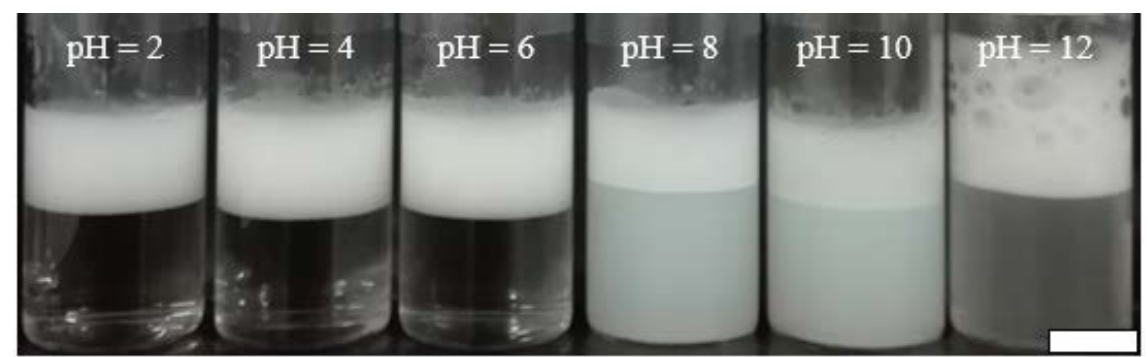

(b)

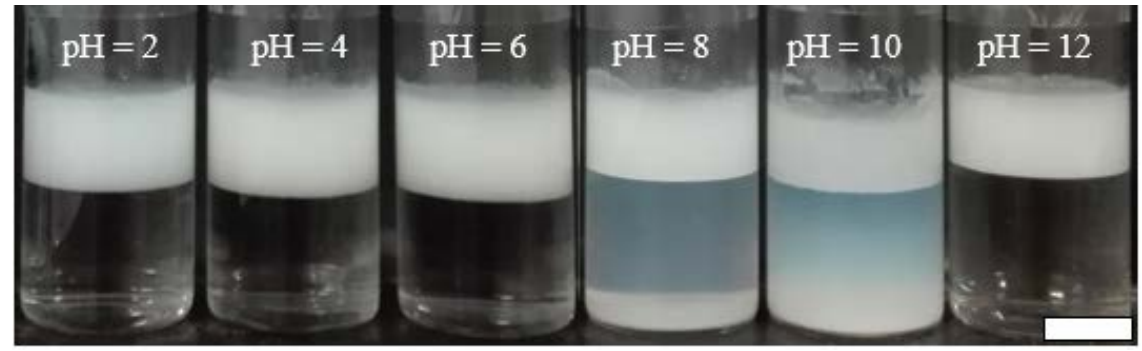

(c) 400

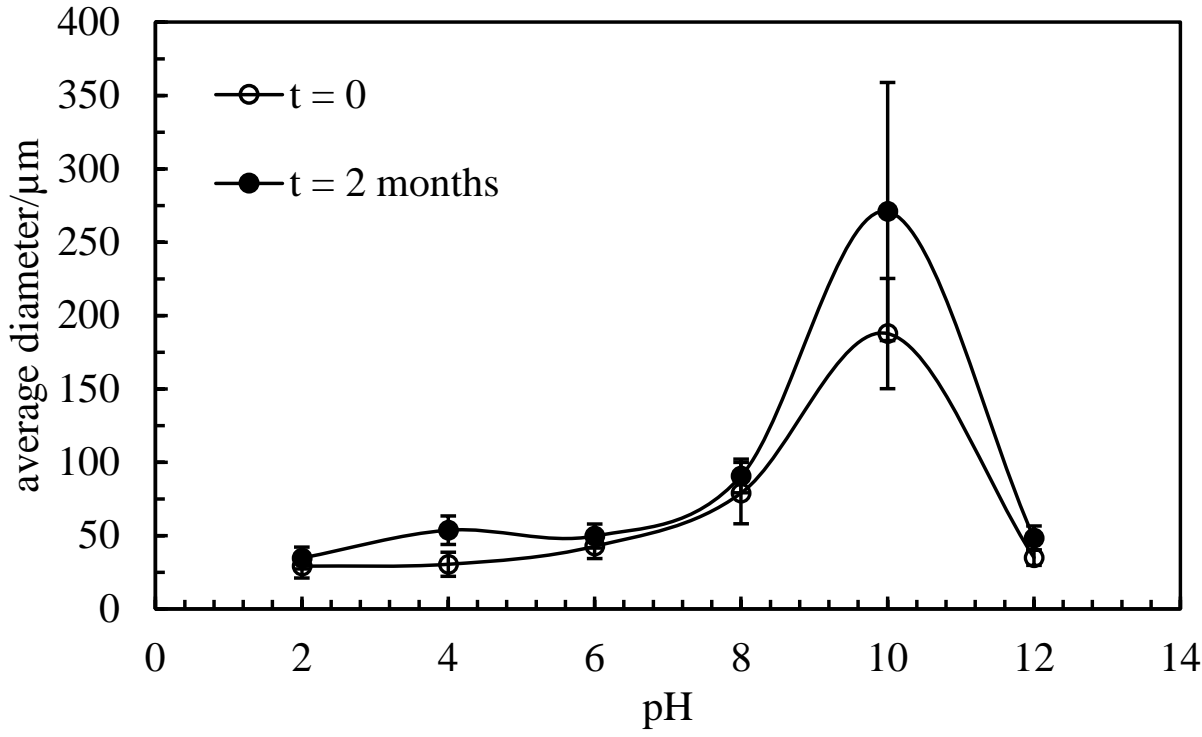


Figure 7. Appearance of emulsions prepared with an aqueous PEC dispersion ( $\mathrm{X}_{\mathrm{PSSNa}}=0.83$, $[\mathrm{PEL}]=10 \mathrm{~g} \mathrm{~L}^{-1}, \mathrm{pH}=2$ ) and $n$-dodecane at different oil volume fractions given (a) $15 \mathrm{~min}$ and (b) 2 months after preparation. Scale bar $=1 \mathrm{~cm}$. (c) Selected optical microscope images at different $\phi_{o}$ (given) taken after preparation.

(a)

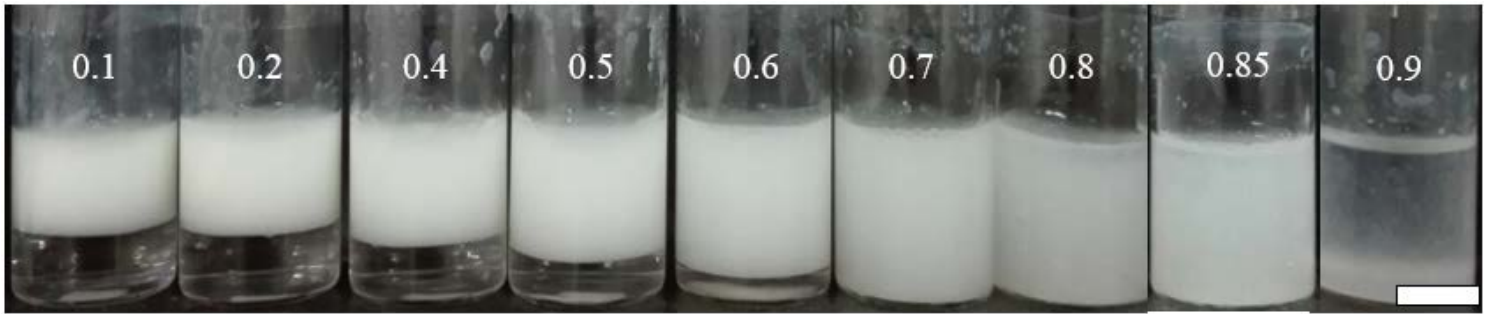

(b)

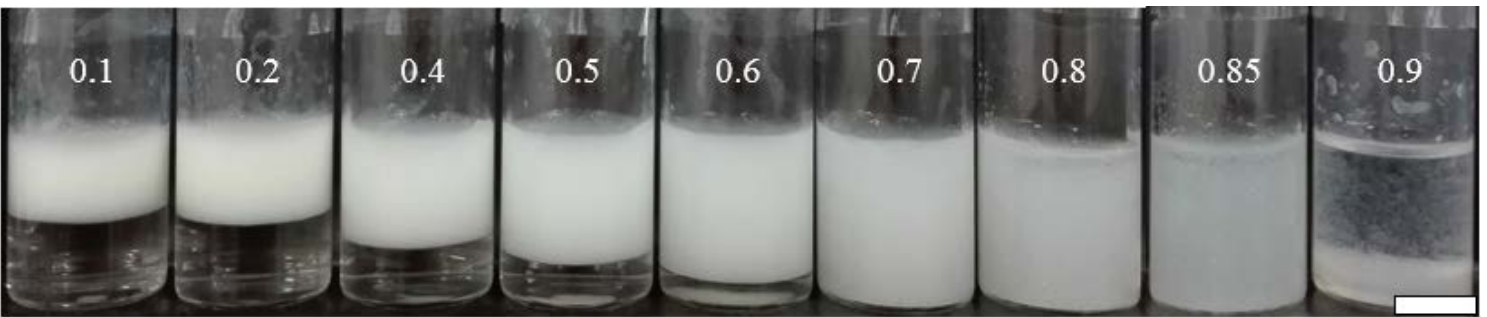

(c)
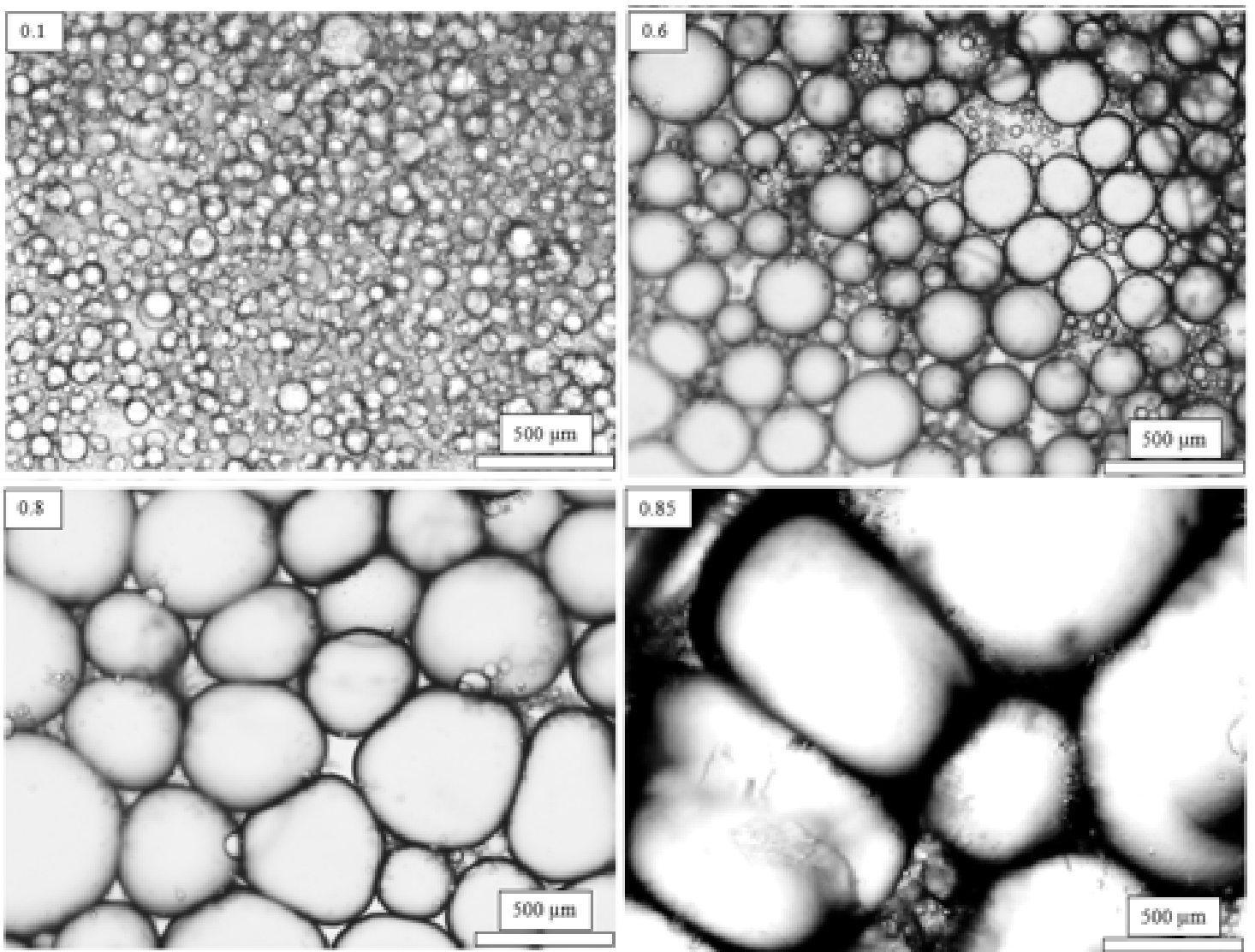
Figure 8. (a) Average droplet diameter versus $\phi_{0}$ for emulsions prepared with an aqueous PEC dispersion (XPSSNa $=0.83,[\mathrm{PEL}]=10 \mathrm{~g} \mathrm{~L}^{-1}, \mathrm{pH}=2$ ) and $n$-dodecane immediately after preparation and 2 months later. (b) Variation of fraction of oil (filled points) or water (open points) resolved after two months versus $\phi_{0}$.

(a)

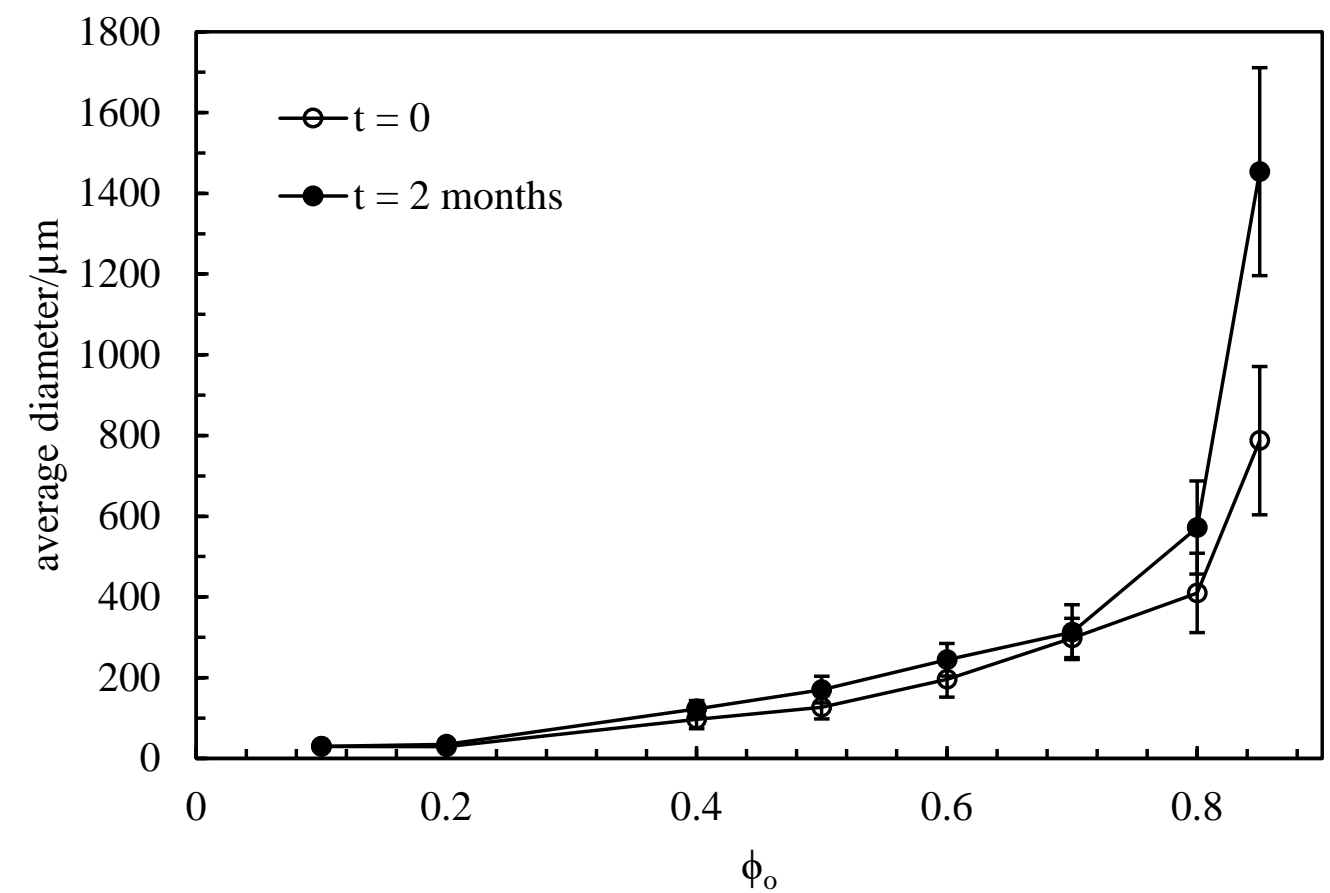

(b)

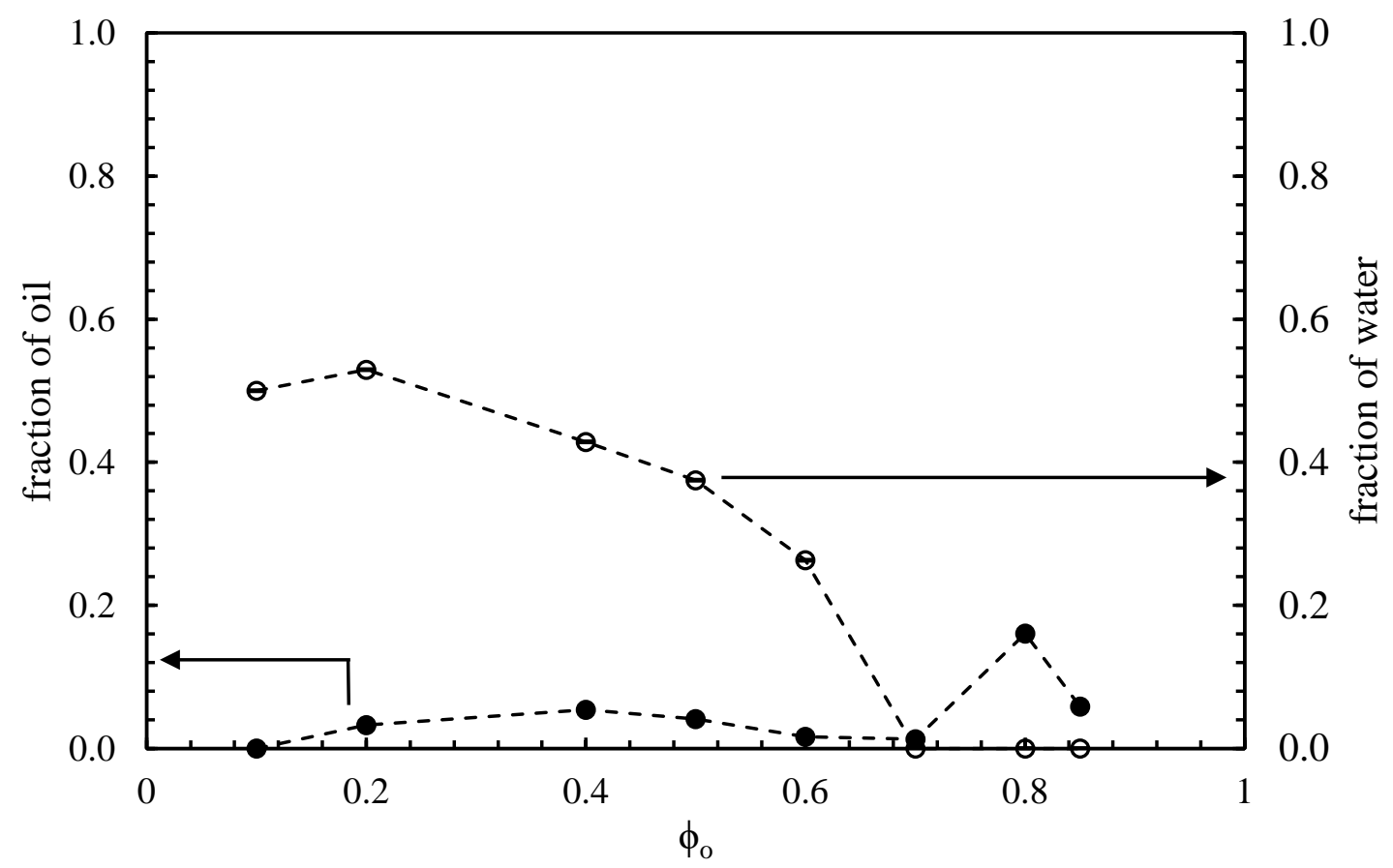


Figure 9. Variation of fraction of oil (filled points) and water (open points) resolved after two months as a function of the initial oil volume fraction for emulsions prepared with $10 \mathrm{~g} \mathrm{~L}^{-1}$ solutions of (a) PAH and (b) PSSNa at $\mathrm{pH}=2$.

(a)

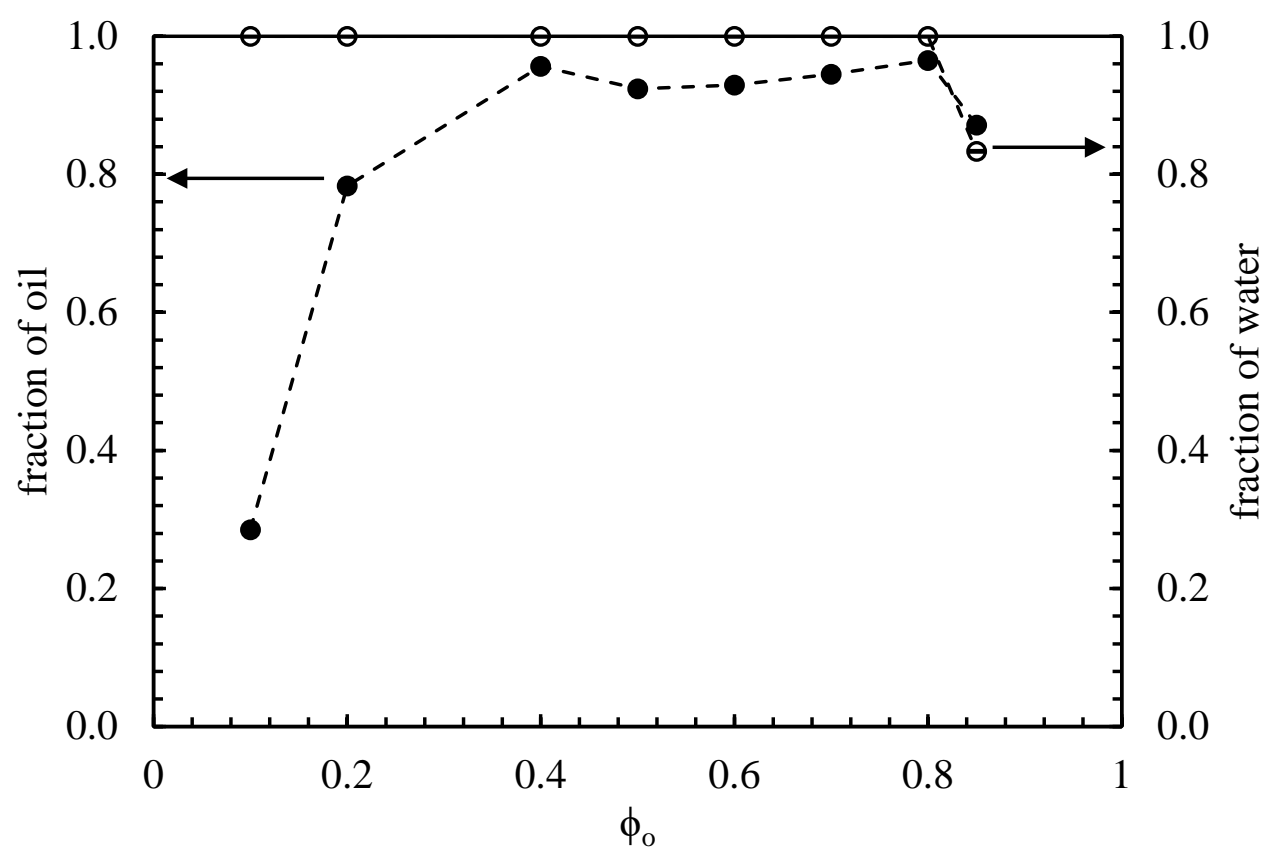

(b)

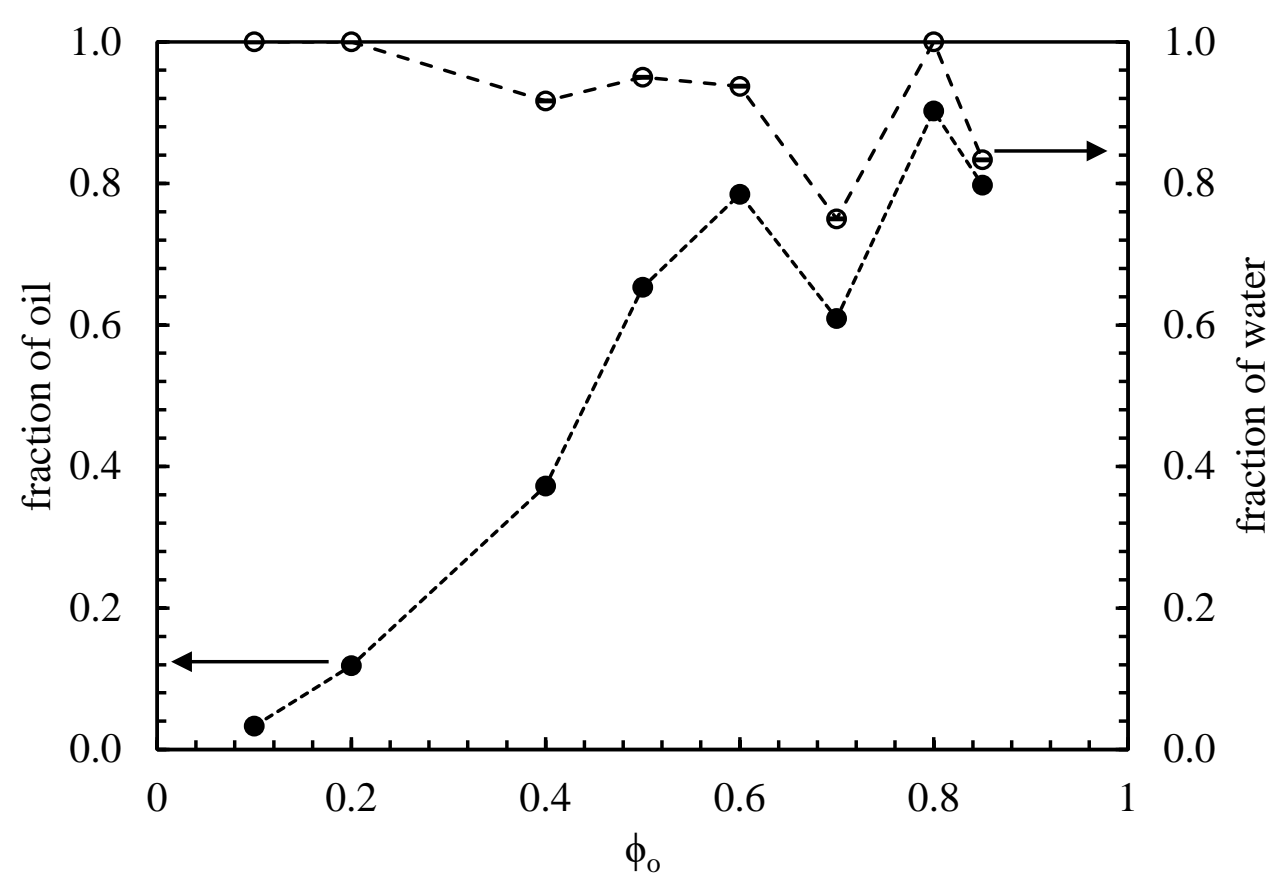


Figure 10. Cryo-SEM images of a freshly prepared emulsion from an aqueous PEC dispersion $\left(\right.$ XPSSNa $=0.83,[\mathrm{PEL}]=10 \mathrm{~g} \mathrm{~L}^{-1}, \mathrm{pH}=2$ ) and $n$-dodecane for (a) $\phi_{o}=0.5$ and (b) $\phi_{o}=0.6$ at different magnifications.

(a)

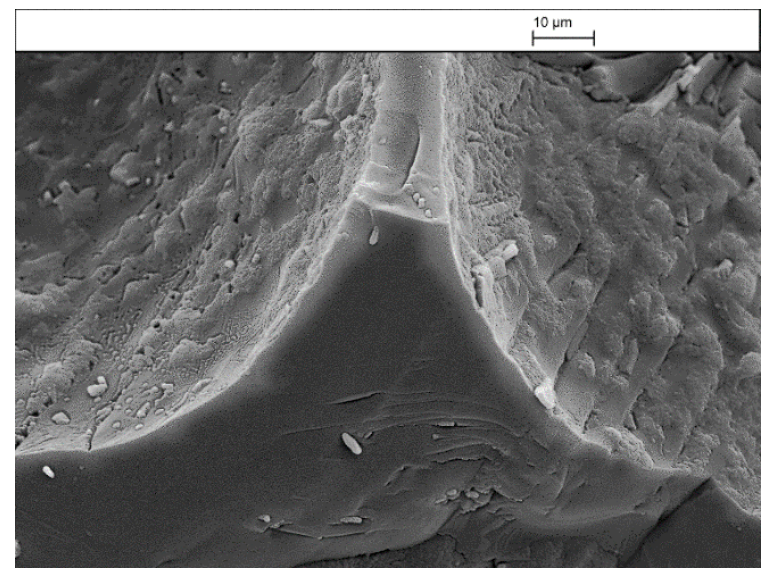

(b)

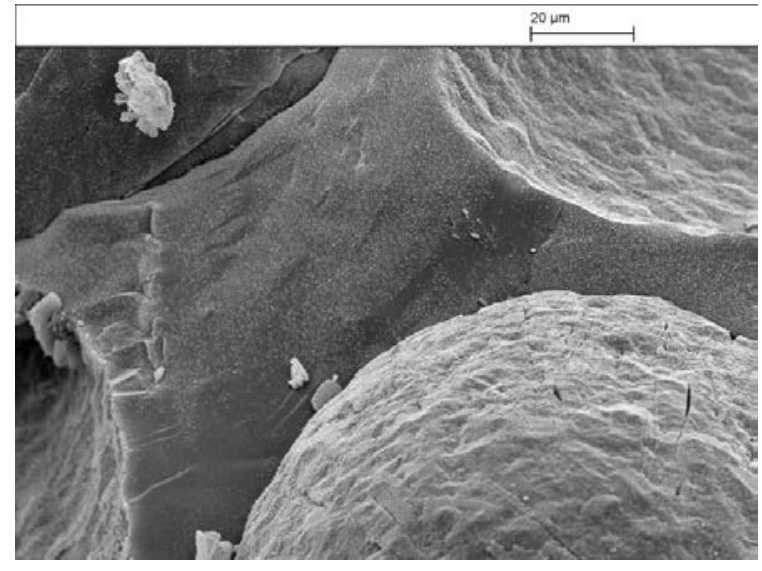

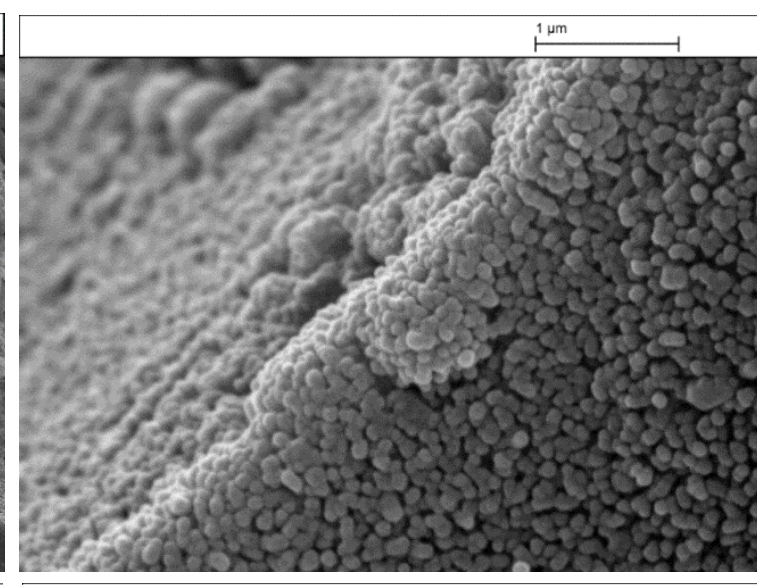

${ }^{2 \mu m}$

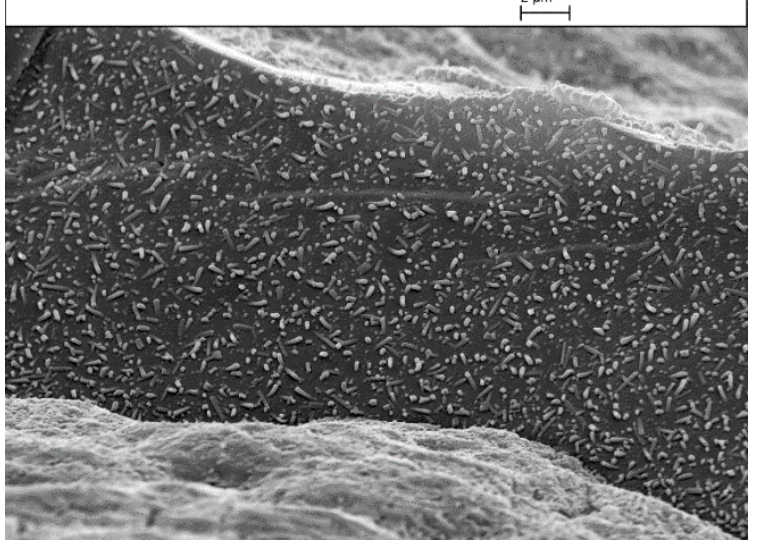


Figure 11. Optical (left) and confocal (right) micrographs of freshly prepared emulsions from an aqueous PEC dispersion (XPSSNa $=0.83$, $[\mathrm{PEL}]=10 \mathrm{~g} \mathrm{~L}^{-1}, \mathrm{pH}=2$ ) and $n$-dodecane at different $\phi_{o}$ : (a) 0.2, (b) 0.6 and (c) 0.8. (d) 3D images for $\phi_{o}=0.8$ taken from different perspectives: $\mathrm{x}$ and $\mathrm{y}=800 \mu \mathrm{m}, \mathrm{z}=400 \mu \mathrm{m}$.

(a)
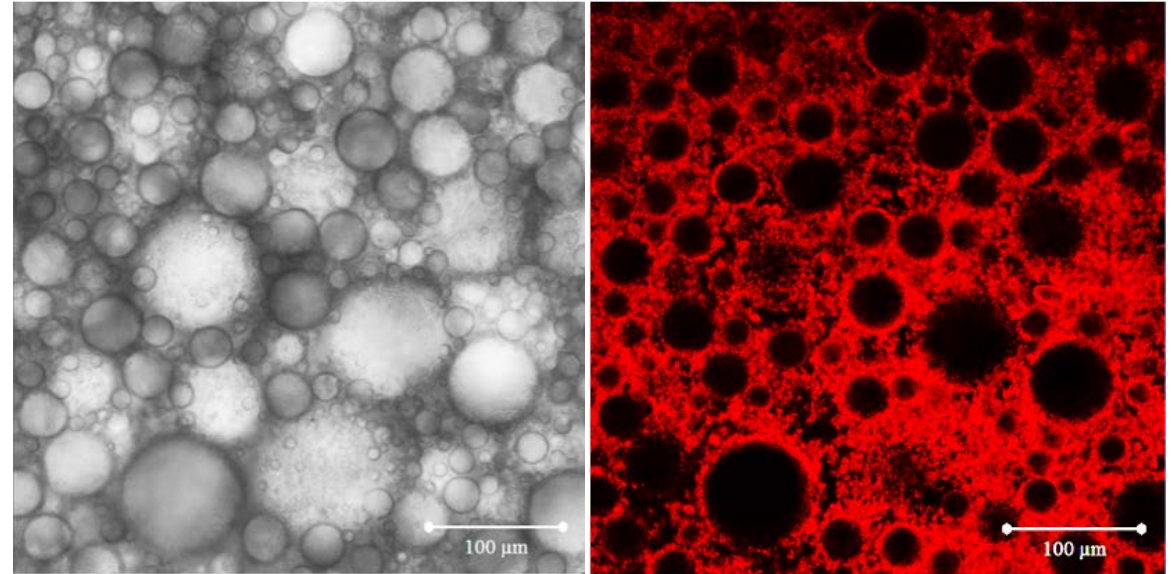

(b)
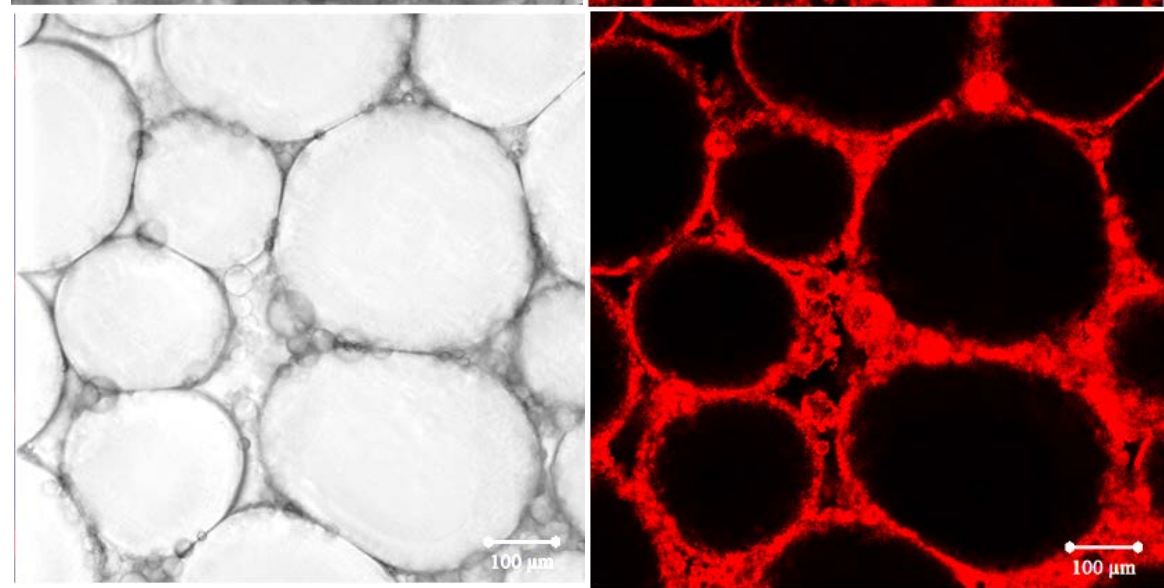

(c)
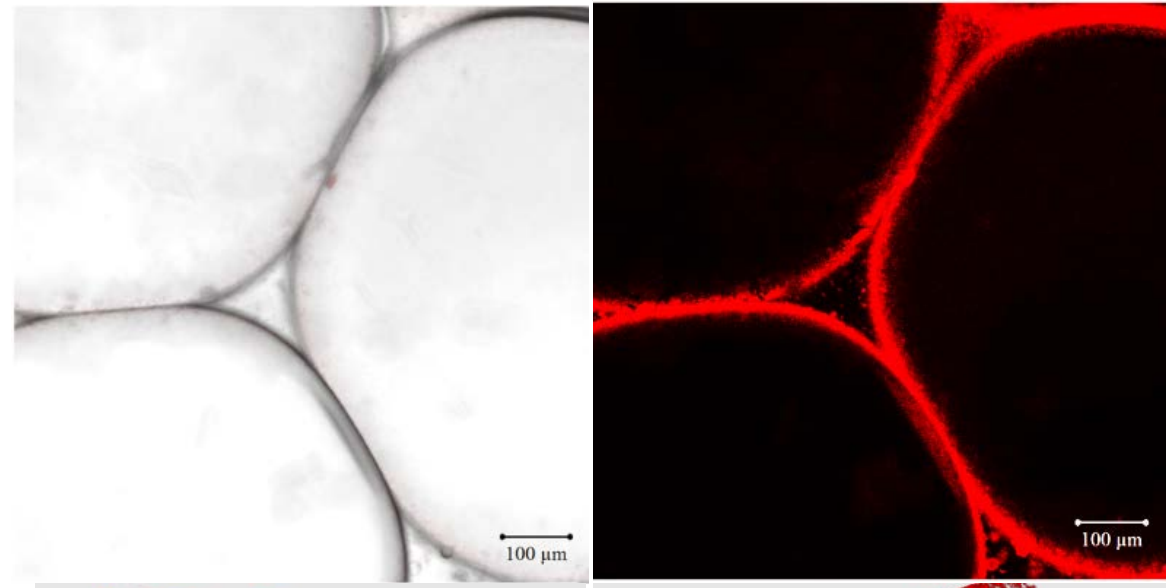

(d)
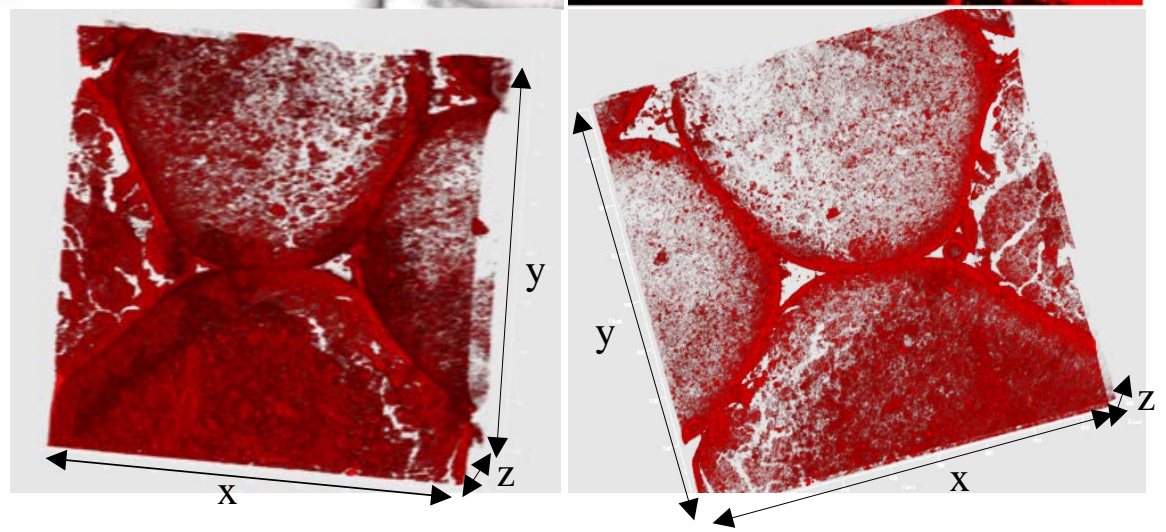
Figure 12. (a) Viscosity versus shear stress for emulsions prepared from aqueous PEC dispersions $\left(\mathrm{X}_{\mathrm{PSSNa}}=0.83,[\mathrm{PEL}]=10 \mathrm{~g} \mathrm{~L}^{-1}, \mathrm{pH}=2\right)$ and $n$-dodecane at different $\phi_{0}$. (b) Viscosity at $\sigma=10$ Pa versus $\phi_{o}$ for the above emulsions.
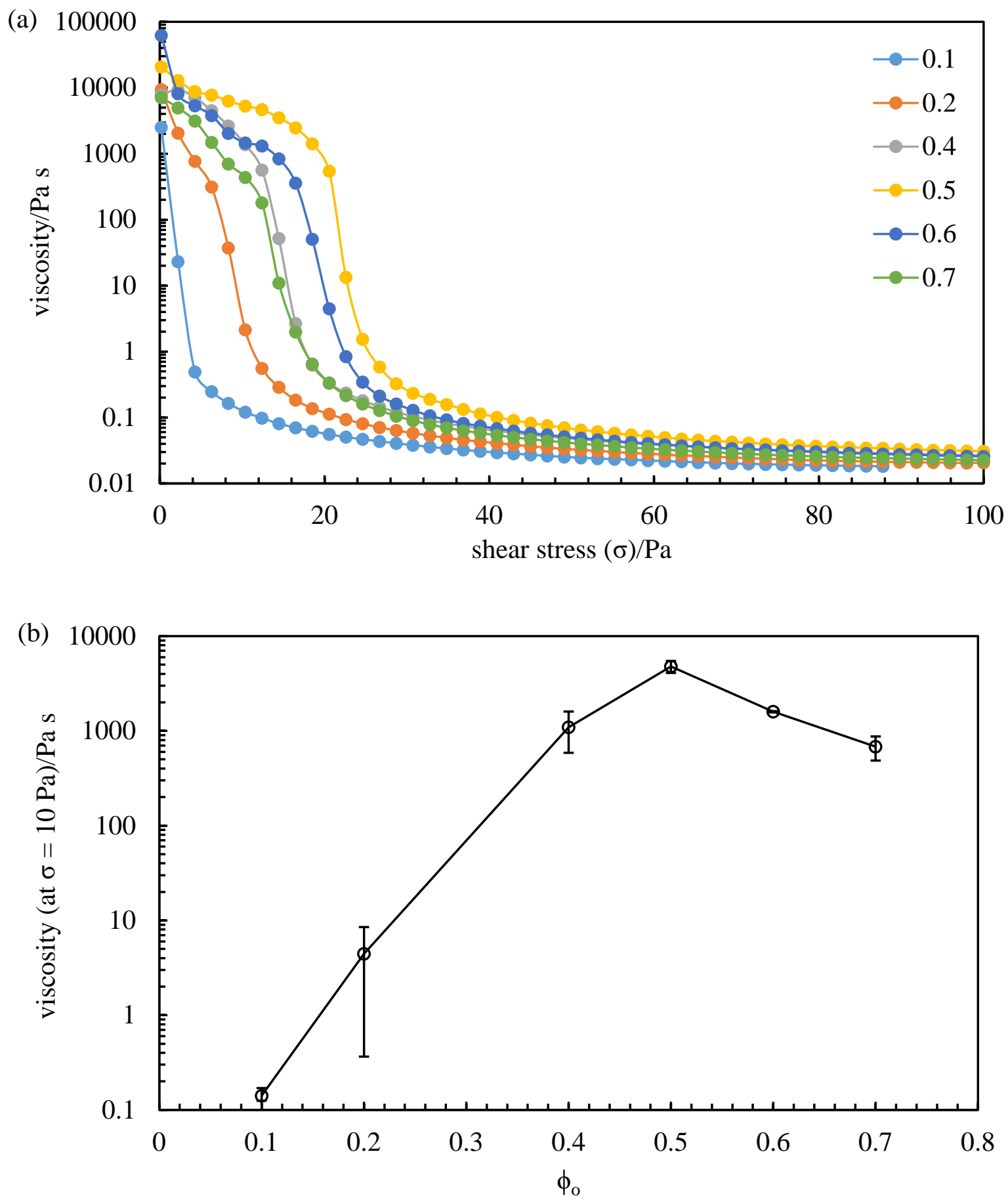